\title{
Posttraumatische Korrekturoperationen am Fuß
}

\author{
Thomas Mittlmeier, Markus Beck
}

\section{Zusammenfassung}

Posttraumatische Korrektureingriffe am Fuß sind dann erforderlich, wenn im Rahmen der primären Behandlungsstrategie Verletzungen oder Teilkomponenten undiagnostiziert bleiben, inadäquat primär therapiert werden oder Komplikationen im weiteren Verlauf eintreten wie Fehlstellungen und Gelenkstufen mit Ausbildung einer posttraumatischen Arthrose, Pseudarthrosenbildung, aseptische Knochennekrosen oder Infektionen. Ziel einer jeglichen Rekonstruktion ist eine möglichst anatomiegerechte Wiederherstellung der Geometrie des Fußes und eine stabile und plantigrade Fußposition als Voraussetzung für eine akzeptable Gehfunktion und ein gutes beschwerdearmes funktionelles Resultat. Eine profunde Kenntnis der Funktionen der anatomischen Strukturen des Fußes bildet die Basis für den Entwurf eines validen Korrekturkonzepts. Die Korrekturoptionen sind vielfältig und umfassen Osteotomien, autologe Knochenspan-/Spongiosaplastiken, reorientierende Arthrodesen, und ein adäquates Weichteilbalancing inklusive Sehnentransfers. Diese zahlreichen Verfahren können miteinander nach den individuellen Erfordernissen kombiniert werden, wobei eine Risikominimierung des Folgeingriffs einen weiteren wichtigen Parameter für den bleibenden Erfolg der Korrektur darstellt.

\section{Post-Traumatic Correction of the Foot}

Post-traumatic correction measures are indicated in case of a primary missing of the diagnosis, the selection of inadequate treatment or in the manifestation of complications in the further clinical course such as, e.g., malalignment or formation of joint steps with consecutive post-traumatic osteoarthritis, non-union, avascular necrosis or deep infection. The principal goal of any reconstruction is to achieve the best possible anatomic reconstruction of foot geometry and a stable and plantigrade foot position as a prerequisite of acceptable gait function and an overall satisfactory and pain-free outcome for the patient. Profound knowledge about the functions of the anatomic foot structures is the basis for the development of a valid therapeutic strategy. The options for correction are manifold and include osteotomies, autologous bone grafting, corrective arthrodeses and a soft-tissue balancing including tendon transfers. These procedures can be combined according to the individual requirements while a minimisation of the perioperative risks represents a further important parameter for the permanently successful procedure.

\section{Problematik}

Verletzungen des Fußes schließen neben knöchernen Läsionen oftmals Gelenkstrukturen und somit auch den je-

OP-JOURNAL 2007; 23: 128-141

(c) Georg Thieme Verlag KG Stuttgart • New York

Am Fuß sind zahlreiche Gelenke funktionell eng miteinander verknüpft.

Gelingt mithilfe unserer primären Behandlungsstrategie keine Heilung in anatomiegerechter Position ist zwangsläufig das funktionelle Zusammenspiel der Strukturen des Fußes, die biomechanisch eng miteinander gekoppelt sind (Abb.1 a), beeinträchtigt.

Die Ursachen hierfür sind mannigfaltig $[17,21,25,26]$. Störungen etwa im Subtalargelenk wirken sich somit erheblich auf die Funktion im Chopart-Gelenk und im oberen Sprunggelenk aus $[1,15]$. Mittels Inversion und Eversion des Rückfußes wird eine Divergenz bzw. Parallelstellung der Achsen von Talus und Kalkaneus bzw. eine phasenhafte Rigidität bzw. Flexibilität des Fußes beim Abrollvorgang gewährleistet. Im Zusammenspiel mit Supination und Pronation des Vorfußes und Innen- bzw. Außenrotation der Tibia resultiert hieraus stets eine plantigrade Fußposition in Abhängigkeit von der lokalen Beschaffenheit des Untergrunds (Abb.1a). Wird diese funktionelle Kette an einer Stelle unterbrochen, resultieren oftmals Abrollstörungen, der Patient hinkt. Ungünstigenfalls liegt der Fuß bereits im Stehen nicht mehr in physiologischer Weise dem Boden auf, d.h. es existiert keine plantigrade Fußposition mehr [12]. Statische knöcherne Fehlstellungen bedingen mittelfristig dynamische Anpassungsvorgänge (etwa der Sehnenstrukturen und -funktionen). Dies zieht neben erheblichen Störungen der Gehfunktion oftmals Fehlbelastungen der Partien mit Bodenkontakt beim Stehen und Gehen und lokale Weichteilprobleme nach sich. Nach komplexem Trauma mit begleitendem Weichteilschaden können schwerwiegende kombinierte ossäre und Gelenkdeformitäten sowie Weichteilprobleme (z.B. Fibrose der kurzen Fußmuskulatur mit Krallenzehenbildung nach übersehenem und/ 
oder inadäquat behandeltem Fußkompartmentsyndrom) resultieren $[12,25]$.

Eine plantigrade Fußstellung ist für die statische und dynamische Funktion des Fußes wichtig.

\section{Topografische Eingrenzungen}

Wir unterscheiden Rückfuß von Mittfuß (Fußwurzel) und Vorfuß. Das obere Sprunggelenk (OSG, „ankle joint“) zählt im engeren Sinn nicht zum Rückfuß. Somit soll das obere Sprunggelenk im Folgenden nur insoweit in die Überlegungen einbezogen werden als es sekundär in eine primäre Störung des USG involviert wird („ankle hindfoot complex“).

Im Bereich des Rückfußes können wir funktionell aufgrund der engen Koppelung die drei Kammern des unteren Sprunggelenks (USG), nämlich das Subtalargelenk, das Talonavikulargelenk und das Kalkaneokuboidgelenk, wie im angloamerikanischen Sprachraum üblich, zusammenfassen, wenngleich wir das vordere untere Sprunggelenk auch als Chopart-Gelenk bezeichnen. Auch das vordere untere Sprunggelenk und die tarsometatarsalen Gelenke (LisfrancGelenke) sind funktionell eng miteinander verbunden [1]. Für die Erfassung und Beurteilung geometrischer Störungen im Bereich des Fußes ist das ZweiSäulen-Modell hilfreich (Abb.1 b): wir unterscheiden hier eine mediale von einer lateralen Fußsäule mit den jeweils dazu gehörigen Strukturen [25,26].

\section{Pathobiomechanik}

Am Fuß differenzieren wir zudem funktionell essenzielle von nicht essenziellen Gelenken [12,19]. Essenzielle Gelenke (z.B. das Talonavikulargelenk, die TMT4- und -5-Gelenke) sollten, wenn immer möglich, in ihrer Funktion bei einer Rekonstruktion erhalten werden. Die Schlüsselrolle für die Funktion des Rückfußes kommt insbesondere dem Talonavikulargelenk („coxa pedis“) zu [10, 12,17,19]. Eine Limitierung des Bewegungsausschlags im Talonavikulargelenk bedeutet eine erhebliche Beschränkung oder gar Aufhebung der Rückfußbeweglichkeit. In-vitro-Studien an Kadaverpräparaten zeigten, dass eine Ausschaltung der Funktion des Talonavikulargelenks eine Restriktion der Beweglichkeit zu 98\% im Subtalargelenk und zu 98\% im Kalkaneokuboidgelenk nach sich zieht [1]. Eine Talonavikulargelenksarthrodese ist somit funktionell

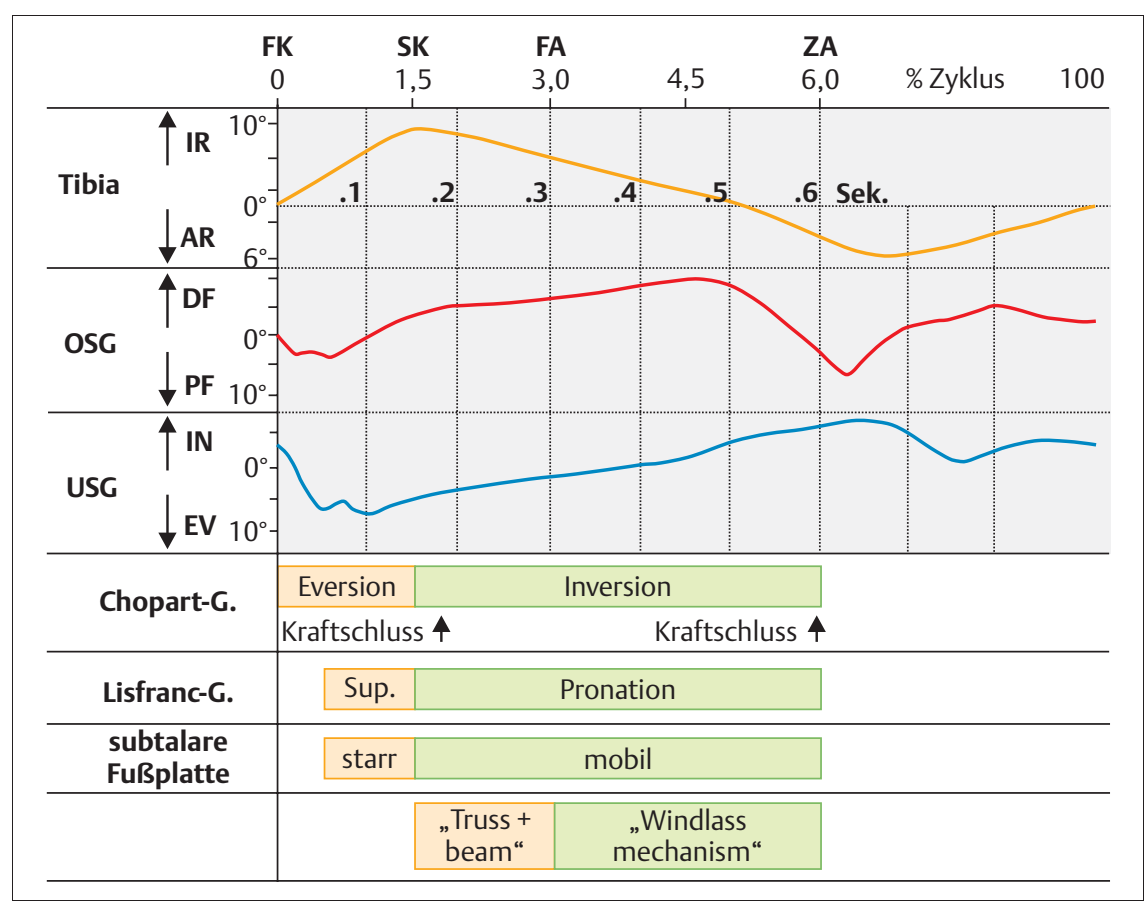

Abb. 1 a OSG, USG und Fußwurzel sind funktionell beim Abrollvorgang gekoppelt.

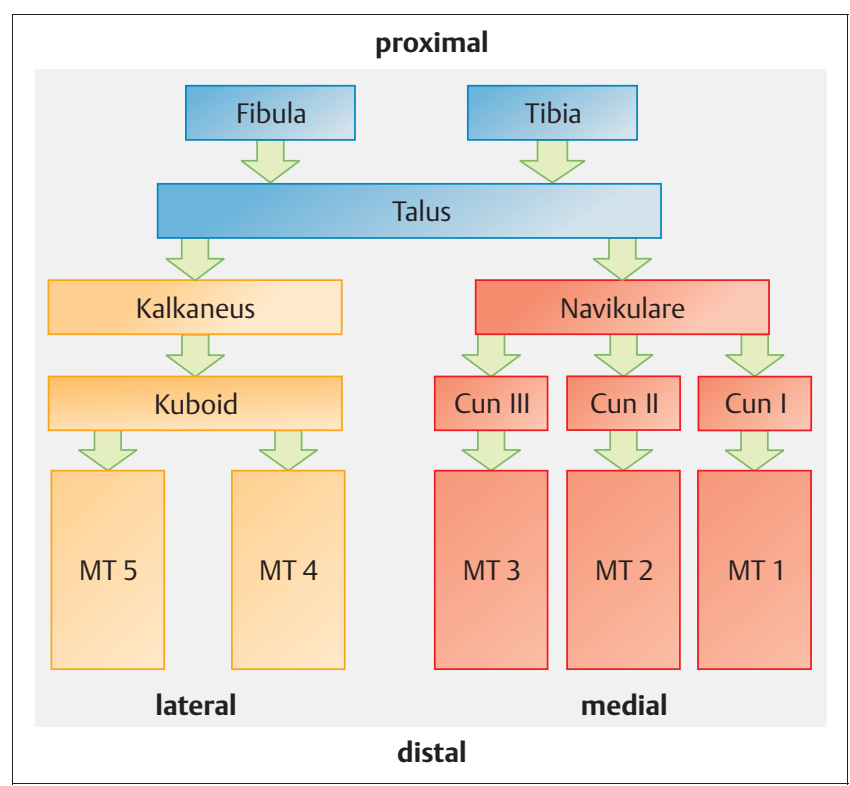

Abb. 1 b Das ZweiSäulen-Modell des Fußes. Geometrie und Funktion beider Fußsäulen sind innig aufeinander abgestimmt.

einer Tripelarthrodese, also der Ausschaltung aller 3 USG-Kompartimente (Subtalargelenk und Talonavikular- und Kalkaneokuboidgelenk), gleichzusetzen. Ein isolierter Verlust der Beweglichkeit im Subtalargelenk nach Arthrodese bedeutet nur eine Einbuße von 74\% im Talonavikulargelenk und von 44\% im Kalkaneokuboidgelenk [1]. Des Weiteren resultiert aus einer isolierten Arthrodese des Kalkaneokuboidgelenks (ein nicht essenzielles Gelenk) eine Funktionsbe- schränkung im Talonavikulargelenk von nur 33\% und im Subtalargelenk von 0\% [1].

Jegliche Arthrodese am Fuß kann also typische funktionelle Beschränkungen der Nachbargelenke und im Langzeitverlauf auch eine Anschlussarthrose nach sich ziehen [17]. Eine Tripelarthrodese beeinträchtigt auch die Funktion des oberen Sprunggelenks durch Limitierung der Extensions- und Flexions- 
beweglichkeit um ca. 15\% [15]; somit können wir nun verstehen, dass eine Tripelarthrodese ähnlich wie die talonavikuläre Arthrodese bei jedem dritten Patienten signifikante arthrotische Veränderungen am OSG beobachten lässt und, wenn möglich, bei einer Rekonstruktion umgangen werden soll.

Bei der Rekonstruktion des Fußes sind Arthrodesen sparsam einzusetzen. Es gibt keine zwangsläufig zu kombinierenden Arthrodesen!

Störend auf die Funktion des Fußes wirken sich auch Achsfehlstellungen des Rückfußes sowie Längendifferenzen der medialen und lateralen Fußsäule aus (Abb.1 b), die wiederum erhebliche Auswirkungen auf das Abrollverhalten haben. Anzustreben ist eine Valgusposition im Rückfuß von 5-10 Grad, um hiermit eine flexible Position der Chopart-Gelenklinie und eine plantigrade Stellung des Fußes beim Abrollvorgang zu erzielen $[12,17]$.

Anzustreben ist eine plantigrade Fußposition und die anatomisch korrekten geometrischen Beziehungen der Fußachsen.

Eine fixierte Rückfußvarusstellung oder eine Supinationsstellung des Vorfußes beding eine Mehrbelastung der lateralen Fußsäule ohne Kompensationsmöglichkeit im OSG: Es resultiert eine schlechte und schmerzhaft beschränkte Gehfunktion. Extraanatomische Rekonstruktionen können als Salvage-Verfahren hilfreich sein, eine annähernd normale Fußfunktion ist jedoch nicht zu erwarten.

\section{Ursachen einer posttraumatischen Fehlstellung}

Häufigste Ursache für die Entwicklung einer posttraumatischen Arthrose des USG sind intraartikuläre Kalkaneusfrakturen oder Talusfrakturen. Sind schwere Knorpelschäden des Subtalargelenks bereits nach dem Trauma festzustellen oder gelingt die anatomiegerechte Rekonstruktion nicht - etwa wenn Gelenkstufen $>1-2 \mathrm{~mm}$ verbleiben - sind starke Beschwerden regelhaft vorhanden und führen zur Indikation von Korrektureingriffen. Extraartikuläre Beschwerdeursachen nach Fersenbeinfraktur können sich oftmals mit intraartikulären überlagern $[2,4,5,6,7,8,10,21,25]$ :
1. durch Verbreiterung der lateralen Fersenbeinwand mit Einklemmen der Peronäalsehnen (Peronäalsehnen-Impingement) zwischen Fibulaspitze und Kalkaneuswand

2. Abstützen der Fibulaspitze bei Rückfußvalgus („abutment“)

3. Dorsalkippung des Talus bis zum knöchernen Kontakt zwischen anteriorer Tibiakante und Talushals mit Beschränkung der Beweglichkeit im OSG und

4. bei Höhenminderung des Fersenbeins mit relativer Insuffizienz der Achillessehne und der Trizepsfunktion mit konsekutiven Abrollstörungen.

Gerade bei Talusfrakturen richtet sich oftmals das Hauptaugenmerk des Behandlers auf das obere Sprunggelenk, wobei das untere Sprunggelenk nicht nur bei Taluskorpusfrakturen, sondern auch bei Talushalsfrakturen direkt oder indirekt beteiligt ist [26]. Unterschätzt hinsichtlich ihres Potenzials zu Funktionsstörungen und/oder einer posttraumatischen Arthrose sind periphere Talusfrakturen, die nicht selten primär übersehen werden [26]. Eine primäre Mitbeteiligung des Kalkaneokuboidgelenks findet sich bei zwei Dritteln aller Kalkaneusgelenkfrakturen, wobei eine symptomatische Arthrose des Kalkaneokuboidgelenks sehr viel seltener zu beobachten ist (vgl. Abb. 2d).

Primäre Verletzungen der Chopart-Gelenklinie zeichnen sich durch eine hohe Varianz des Verletzungsmusters aus, wobei ligamentäre und osteokartilaginäre Verletzungen häufig miteinander kombiniert sind [20]. Rasanztraumata, insbesondere Crushverletzungen, enden häufig in einer symptomatischen Arthrose der ventralen Anteile des USG, wobei neben dem primär traumatischen Knorpelschaden oder Gelenkstufenbildungen (Inkongruenzarthrose) resultierende oder verbleibende Fehlstellungen der Fußsäulen und ligamentäre Instabilitäten ihren bleibenden Beitrag leisten (vgl. Abb.1 b). Lisfranc-Gelenkverletzungen können ebenfalls Folge eines Hochgeschwindigkeitstraumas und mit Chopart-Gelenkläsionen vergesellschaftet sein (bis zu einem Drittel der Fälle $[23,25])$. Lisfranc-Gelenkläsionen zeigen allerdings auch eine große Variabilität und finden sich auch nach Sportverletzungen (meist als rein ligamentäre Läsionen). Werden diese primär übersehen (bis zu $25 \%$ !), resultiert unweigerlich eine Arthrose im betroffenen Abschnitt der Fußwurzel und konsekutiv eine Fehlstellung [12,16,17,25]. Fehlstel- lungen am Vorfuß sind oftmals nach Kettenverletzungen der Metatarsalia zu finden. Zehenfehlstellungen sind $\mathrm{zu}-$ meist weniger Folge eines direkten Traumas, sondern Zeichen eines abgelaufenen schweren Weichteiltraumas mit Sehnenkontrakturen der langen und kurzen Beuger bzw. der intrinsischen Muskeln und führen bei fixierter Fehlstellung zur Funktionslosigkeit der entsprechenden Zehenstrahlen und zum Schuhkonflikt [12,19].

\section{Diagnostik}

Eine vollständige Erfassung der Anamnese und der individuellen Schmerzund Funktionsstörungen des jeweiligen Patienten ist Voraussetzung einer Korrekturplanung.

Hierzu gehört auch eine Auflistung der bisherigen therapeutischen Maßnahmen.

\section{Klinik}

Eine Auflistung sämtlicher klinischer Tests und Untersuchungsmethoden würde den Rahmen dieser Arbeit sprengen. Die Erfassung instabiler Narben, chronischer Schwellungszustände, vorbestehender Narbenfelder oder zuvor erfolgter plastischer Deckungsmaßnahmen sind relevante Parameter für die spätere Zugangswahl [6]. In jedem Fall sind neben dem üblichen Algorithmus der klinischen Untersuchung (Inspektion, Palpation) die aktiven und passiven Gelenkbeweglichkeiten zu prüfen. Insbesondere bei monolateralen Verletzungen ist der systematische Vergleich mit der unverletzten Seite hilfreich. Auch die orientierende klinisch-angiologische (Zeichen der chronisch-venösen Insuffizienz, Fußpulse) und neurologische Untersuchung (sensomotorische Defizite) ist elementarer Bestandteil der kompletten Erfassung des aktuellen Status vor Korrektur.

Die Untersuchung im Stehen am bis auf die Unterwäsche entkleideten Patienten erlaubt neben der Erfassung von Beinlängendifferenzen, die Beurteilung einer Spitzfußkomponente und die Beurteilung der Bein- und Fußachsen unter Last. Der Winkel zwischen der Achillessehne und der Kalkaneusvertikalachse beträgt üblicherweise 5 - 7 Grad Valgus $[8,12,25]$. Bei mobilem USG rotiert die Ferse im Zehenstand in eine Varusposition [13]. Die kranialen Nachbargelenkfunktionen, also die Beurteilung von 


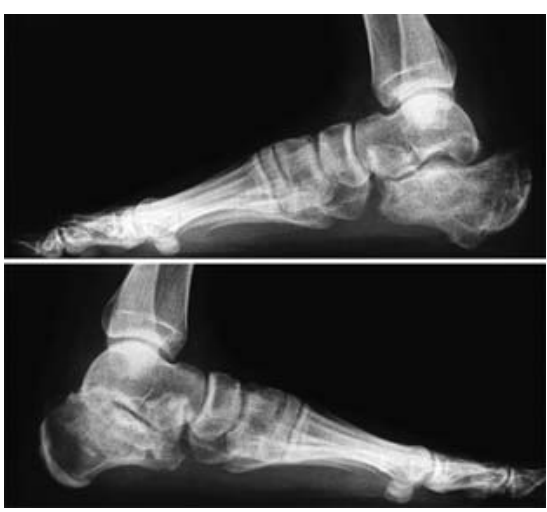

Abb. 2 a 24-jähriger Student; Z. n. beidseitiger intraartikulärer Kalkaneusfraktur in Australien und primär funktionell-konservativer Therapie. Gehstrecke $<100 \mathrm{~m}$. Erstvorstellung 3,5 Monate nach Trauma. Seitliche Aufnahmen beidseits im Stehen. Das Subtalargelenk rechts ist vollständig destruiert, der Böhler-Winkel aufgehoben (unten).

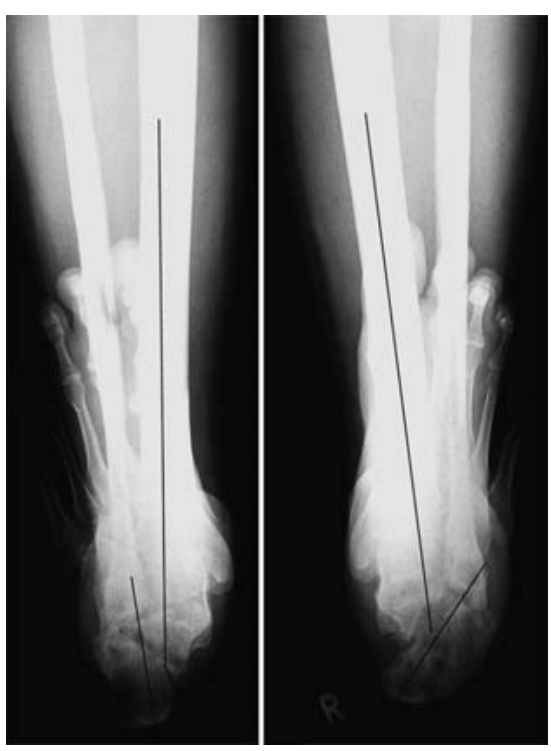

Abb. 2b Saltzman-Aufnahmen der RückfußUnterschenkelachsen beidseits im Stehen. Deutliche Rückfußvarusfehlstellung rechts größer links.

OSG, Knie und Hüfte sind relevant für die Bewertung primärer Störungen und sekundärer kompensatorischer Anpassungsvorgänge. Ipsilaterale Fehlstellungen können allein durch die Untersuchung des gesamten Beines erkannt werden; somit können Fehlschläge vermieden werden, die auf einer alleinigen Betrachtung des Fußes gründen. Etwa bei gleichzeitiger Rückfußdeformität und Genu varum wird die isolierte Rückfußkorrektur nicht erfolgreich sein.

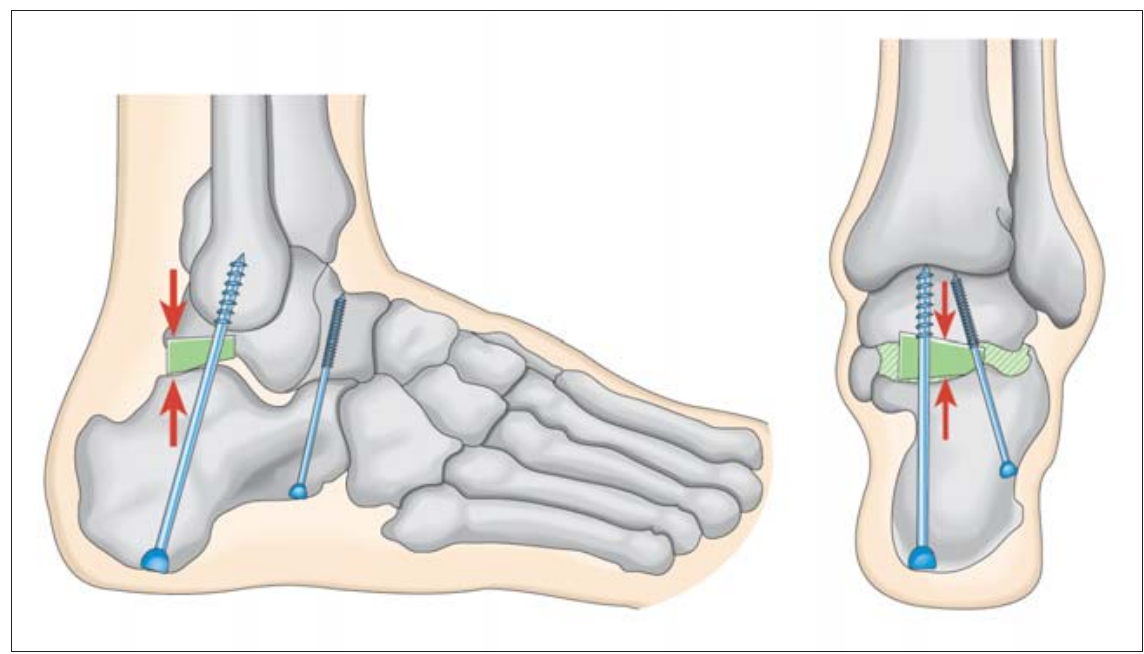

Abb. 2c Prinzip der Korrekturarthrodese im Subtalargelenk nach intraartikulärer Kalkaneusfraktur mit autologem trikortikalem Beckenkammspan und kanülierter 7,3-mm-Schraube bzw. 3,5-mm-Antirotationsschraube.
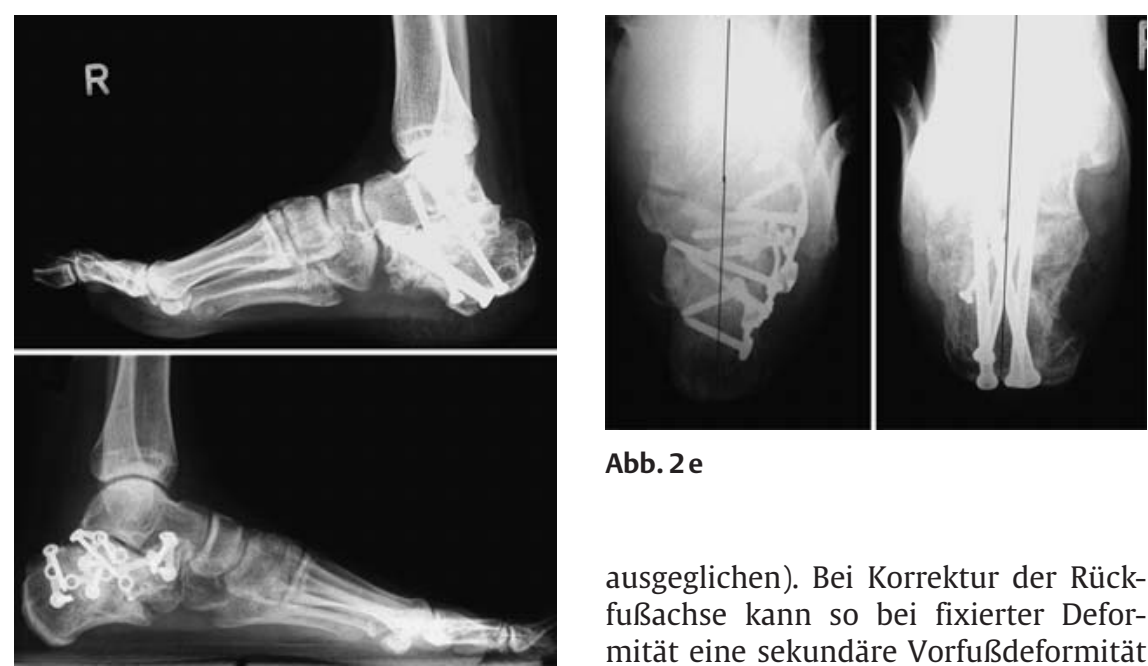

Abb. $2 \mathrm{e}$

Abb. 2d 7 Monate nach Spätrekonstruktion und Osteosynthese links und Korrekturarthrodese links. Belastungsaufnahmen seitlich und Saltzman-Aufnahme beidseits. Die Rückfußvarusfehlstellung wurde beidseits ausgeglichen. Unbegrenzte Gehstrecke des Patienten, er trägt Konfektionsschuhwerk.

Rotatorische Fehlstellungen des Vorfußes können am besten am sitzenden Patienten mit manueller Fixation des Rückfußes in neutral- oder leichter Valgusposition [13] erkannt werden. Entscheidend vor Korrektureingriffen ist die sichere Kenntnis, inwieweit Fehlstellungen des Rückfußes fixiert sind. Bekanntermaßen entwickeln Patienten mit deutlicher Rückfußvalgusdeformität eine kompensatorische Supination im Vorfuß, um eine plantigrade Fußstellung zu erreichen (analog wird ein Rückfußvarus durch Pronation im Vorfuß ausgeglichen). Bei Korrektur der Rückfußachse kann so bei fixierter Deformität eine sekundäre Vorfußdeformität mit Korrekturbedarf resultieren. In gleicher Weise wird eine Hyperpronation im Vorfuß durch eine Inversion des Rückfußes, eine Supination des Vorfußes durch eine Eversion des Kalkaneus ausgeglichen.

Ist eine eindeutige Zuordnung einer Schmerzsymptomatik zu einem spezifischen Gelenk des Fußes nicht praktikabel, kann die Injektion eines Lokalanästhetikums (am besten unter BV-Kontrolle) eine zuverlässigere Zuordnung von Schmerz und Struktur ermöglichen.

\section{Radiologische Diagnostik}

Die Standardröntgenuntersuchung vor einer Korrektur soll Belastungsaufnahmen beider Füße und des OSG im Seitenvergleich im Stehen umfassen: seitlich, dorsoplantar (d.p.) und die lange p.-a. Aufnahme des Fußes und des Un- 
terschenkels nach Saltzman (Abb.2b und d) [22]. Zur adäquaten Beurteilung des OSG ist ergänzend eine Aufnahme des OSG a.-p. mit 20 Grad Innenrotation des Fußes (sog. Mortise-View) erforderlich.

Für die Beurteilung des Chopart-Gelenks ist weiterhin eine 45-Grad-Schrägaufnahme der Fußwurzel (lateral angehoben) nötig. Um den Zentralstrahl der Röntgenröhre optimal auf die interessierende Struktur zu richten, ist bei Störungen im Chopart-Gelenk bei der d.p. Aufnahme eine Kippung der Röhre um 30 Grad nach kaudal, bei Läsion im Lisfranc-Gelenk um 20 Grad zur Vertikalen einzustellen $[23,25]$.

Ganzbeinaufnahmen im Stehen sind wichtig, falls eine Fehlstellung in benachbarten Gelenken oder Segmenten des Beines klinisch vermutet wird: Die Auswertung erfolgt nach den standardisierten Vorgaben von Dror Paley [14].

Zur Analyse komplexer Fehlstellungen und der Arthrose in den Gelenken des Rückfußes und der Fußwurzel ist eine Computertomografie beider Füße inklusive 2D-Rekonstruktion in der axialen und koronaren Ebene angebracht. 3D-Rekonstruktionen als reine Bildinformation sind für die OP-Planung selten hilfreich, die 3D-Datensätze können jedoch mit einer entsprechenden OP-Planungssoftware weiter bearbeitet werden.

Kernspintomografien des Fußes sind im klinischen Gebrauch für die Frage der Arthrose- und Fehlstellungsbeurteilung entbehrlich, bei der gezielten Suche nach Talus- und Navikulareosteonekrosen und Weichteilpathologien (z. B. chronische Osteitis) wertvoll.

\section{Korrekturprinzipien}

Das Ausmaß einer manifesten Fehlstellung oder klinischer Beschwerden nach einer Verletzung des Fußes und das Versagen bisheriger (nicht operativer) therapeutischer Maßnahmen oder die Aussicht, dass nicht operative Maßnahmen nicht Erfolg versprechend sind, bilden die Grundlage für eine Erwägung operativer Korrekturoptionen. Selbstverständlich erscheint es wünschenswert, Gelenk erhaltend Korrekturen - wie auch an anderen großen Gelenken der unteren Extremität - vorzunehmen. Allerdings ist nur in einer relativ frühen Phase nach Trauma (meist wenige Wochen

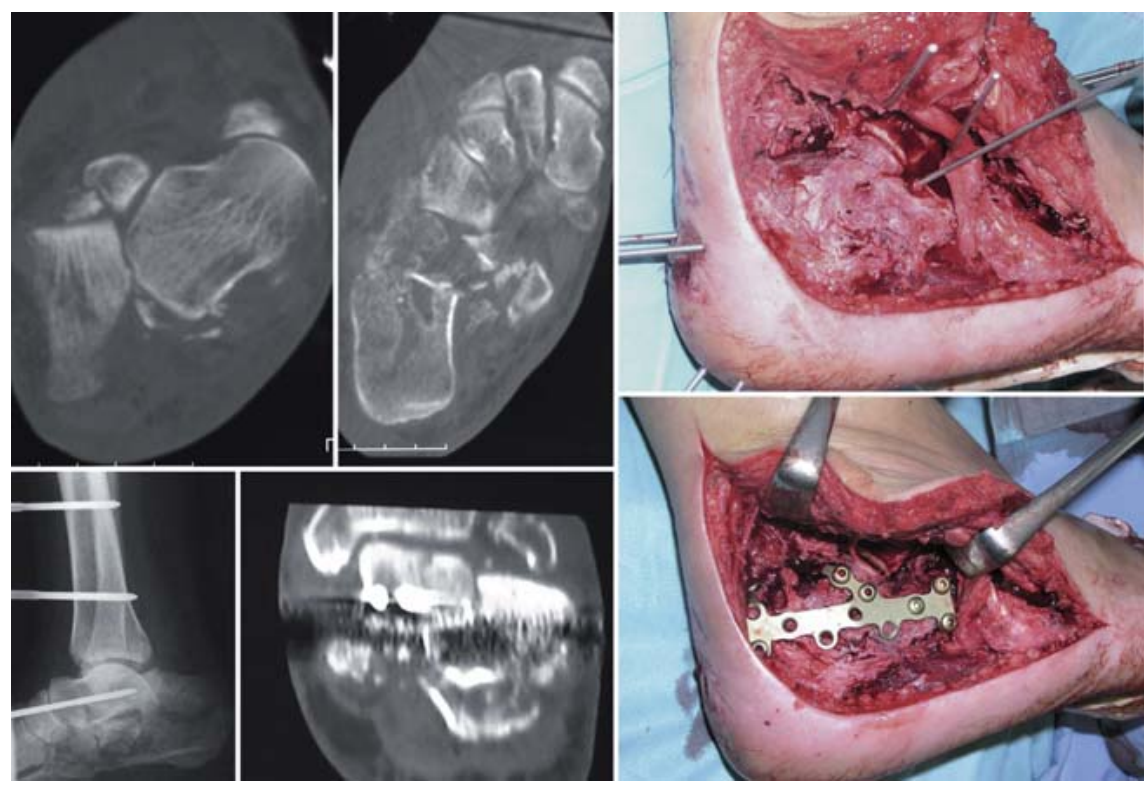

Abb. 3 a 48-jährige Patientin nach Polytrauma u.a. mit Kalkaneusluxationsfraktur. Axiale CTSchnitte im Rahmen der Primärdiagnostik (links oben). Initiale Notfallversorgung mit geschlossener Reposition und tibiometatarsaler Transfixation (links unten). Trotz zusätzlichem Taluspin (Schanz-Schraube) zeigt die koronare Rekonstruktion des Subtalargelenks eine erhebliche Fehlstellung (Mitte unten), die äußere Fersenbeinwand steht lateral der Außenknöchelspitze. Postprimäre offene Rekonstruktion über erweiterten lateralen Zugang (intraoperativer Situs rechts) und Stabilisation mit Kalkaneusplatte und temporärer K-Draht-Transfixation des Subtalar- und Talonavikulargelenks für 6 Wochen.
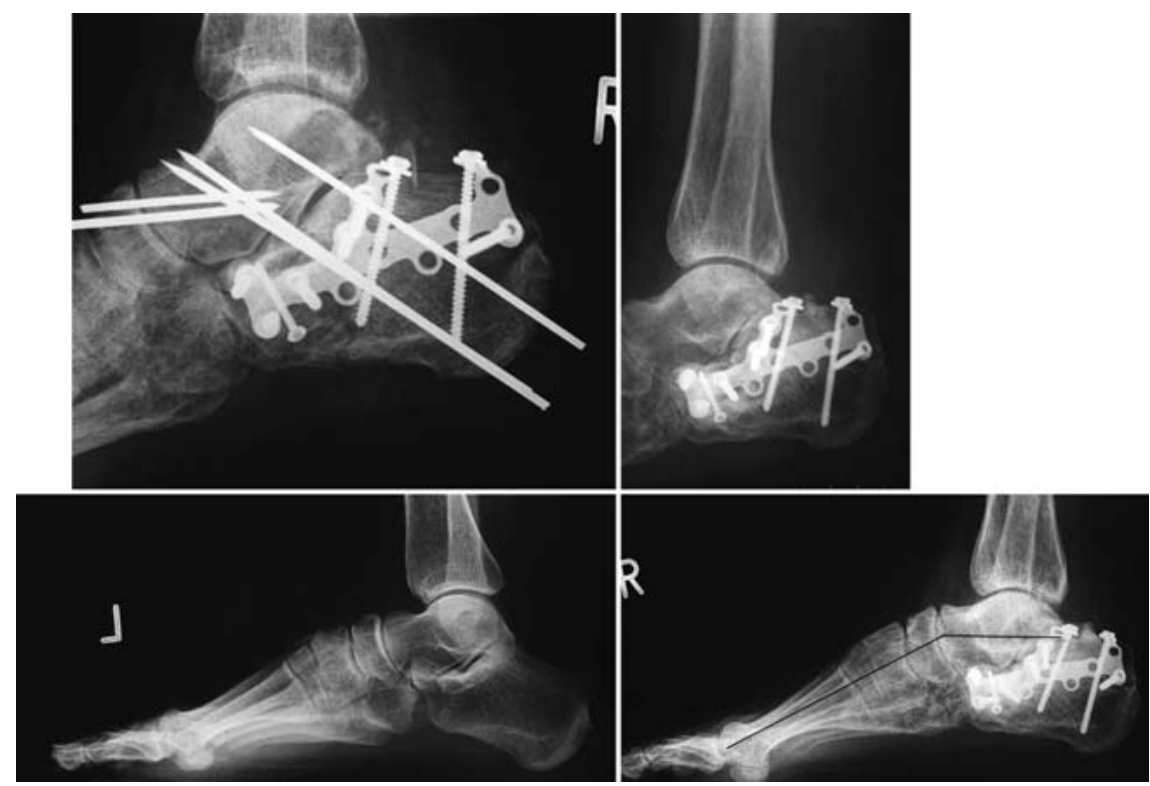

Abb. 3b Postoperatives seitliches Röntgenbild (links oben) und 8 Monate postoperativ; es findet sich ein aufgehobener Gelenksspalt subtalar im Sinne der fortgeschrittenen posttraumatischen Arthrose. Belastungsaufnahmen des unverletzten (links) und verletzten Fußes inkl. OSC mit Subluxation des Talus nach dorsal und Anschlussarthrose im Talonavikulargelenk. Beachte die Spitzfußfehlstellung.

und Monate) bei spezifischen Gelenkverletzungen mit einer erfolgreichen anatomischen Sekundärrekonstruktion zu rechnen. Dies betrifft zumeist Talusfrakturen, seltener intraartikuläre Kalkaneusfrakturen [26] und Verletzungen der medialen Fußsäule wie Navikularefrakturen (Abb.2). Erfolg versprechend sind hier v.a. Taluspseudarthrosen, die vollständig ausgeräumt bzw. reseziert und mit autologen Knochenspan/-spongiosaplastiken aufgefüllt und adäquat 


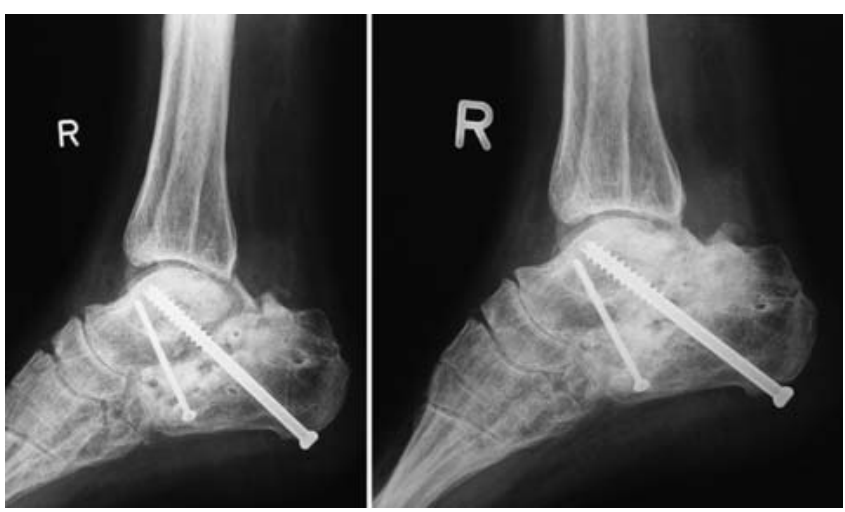

Abb. 3c Subtalare Korrekturarthrodese (Bilder postoperativ und nach 4 Monaten).
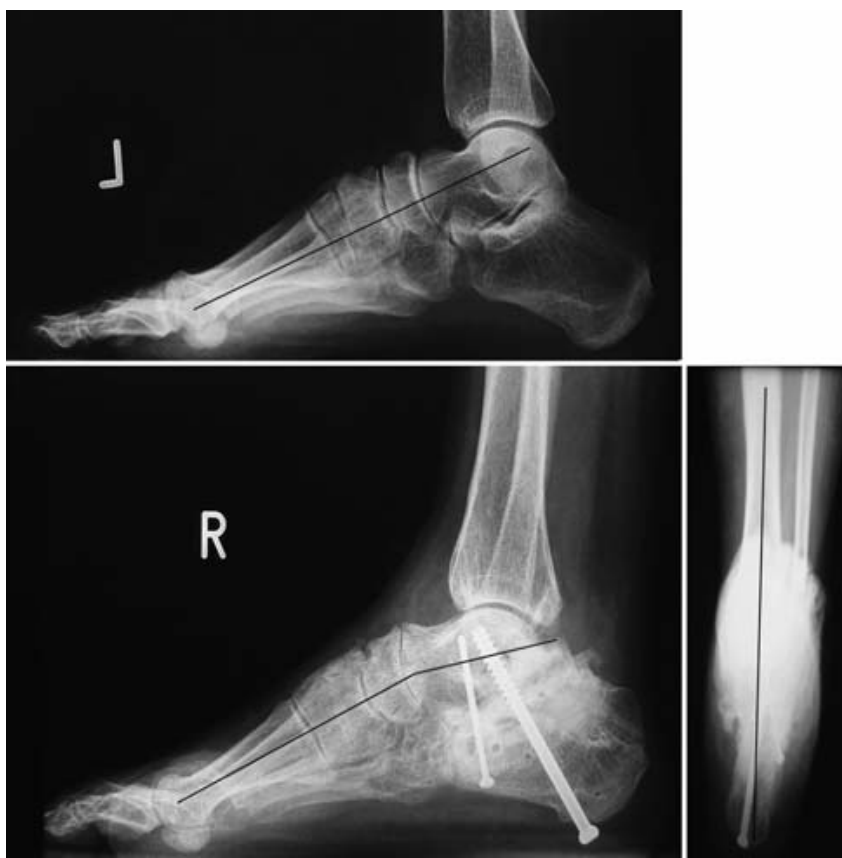

Abb. 3d Belastungsaufnahmen im Vergleich mit der unverletzten Seite; bei Ausgleich der Equinusfehlstellung besteht noch eine residuale Verwerfung der Talo-Metatarsale-I-Achse im Sinne der inkompletten Aufrichtung des Talus.

stabilisiert werden sollten [26]. Mit Fortschreiten einer posttraumatischen Arthrose und Fixierung einer entsprechenden Fehlstellung werden umschriebene Korrekturarthrodesen des Fußes das Korrekturwerkzeug der Wahl. Für die häufig zu beobachtenden Fehlverheilungen des Kalkaneus gibt es eine eigene Einteilung [26].

In-situ-Arthrodesen als alleinigem Instrument der Korrektur sind selten praktikabel, da nur die Korrektur der gleichzeitig vorhandenen Fehlstellung, somit die Korrekturarthrodese oder reorientierende Arthrodese im Sinne der geometrischen Rekonstruktion des Fußes eine Wiederherstellung der Fußachsen und eine plantigrade Fußstellung ermöglicht.
Allein der Verlust der Knorpeldicke bei der In-situ-Arthrodese verändert das Alignement des Fußes. Gravierende Fehlstellungen können nicht allein als knöcherne Korrektur geplant werden; hierzu sind weitere Maßnahmen des Weichteilbalancing wie ein ligamentäres Release und ggf. Sehnenverlängerungen, -straffungen erforderlich. Sehnentransfers als alleinige oder begleitende Maßnahme sind auch ein Instrument der Korrektur bei umschriebenen moto-

Ebenso wie auch an anderen Lokalisationen der unteren Extremität erfolgt die Korrektur regelhaft von proximal nach distal; also wird die Korrektur der Rückfußdeformität vor der Korrektur des Vorfußes stattfinden. rischen Ausfällen.
Komplexe oder kombinierte Fehlstellungen bedürfen einer am besten schriftlich niedergelegten Behandlungsstrategie („master plan“); ferner ist zu prüfen, inwieweit eine ein- oder mehrzeitige Korrektur erforderlich ist, um das Ziel der Korrektur zu verwirklichen.

Extraanatomische Rekonstruktionen sind üblicherweise Salvage-Eingriffen vorbehalten und können v.a. dann erfolgreich sein, wenn alternativ ein Verlust oder Teilverlust des Fußes zu befürchten ist.

\section{Korrektur unter Gelenkerhalt}

Voraussetzung einer erfolgreichen Rekonstruktion unter Gelenkerhalt ist die Überprüfung, inwieweit das Ausmaß des primären Unfallschadens und der eventuell eingetretenen sekundären Schäden die Maßnahme als erfolgreich erscheinen lässt. Eine erweiterte präoperative bildgebende Diagnostik (s.o.) kann hier eine wesentliche Hilfe bieten neben individuellen Parametern wie dem Alter und dem körperlichen Leistungsanspruch des Patienten. Das Ergebnis der Gesamtschau kann am selben Patienten zu unterschiedlichen Resultaten führen (Abb.2): Das Ausmaß der Destruktion der subtalaren Gelenkfläche bei beidseitiger Kalkaneusfraktur mit erheblicher Fehlstellung nach beinahe 4-monatigem konservativen Behandlungsversuch erlaubte bei einem sportlichen 24-jährigen Studenten die Rekonstruktion des Fersenbeins mit Erhalt der subtalaren Facette links, während die fast vollständige Zerstörung des rechten Subtalargelenks hier nur eine subtalare Korrekturarthrose zuließ. In jedem Fall wurde die massive geometrische Fehlstellung aufgehoben (Abb.2d); der zuvor nur noch limitiert gehfähige junge Patient berichtete nach Rekonstruktion und Ausheilung über eine unbegrenzte Gehstrecke und eine limitierte Sportfähigkeit.

\section{Subtalare Arthrodese}

Der Zugangsweg wird davon bestimmt, ob eine einzeitige Implantatentfernung (meist Implantate, die von lateral über einen erweiterten Zugang eingebracht wurden) erfolgen soll. Ansonsten liegen klare Vorteile für einen posterolateralen Zugang auf der Hand, der gleichermaßen in Seitenlage und in Bauchlage (vorteilhaft für die intraoperative Beurteilung der Rückfußachse) des Patienten angegangen werden kann. Eine verbrei- 


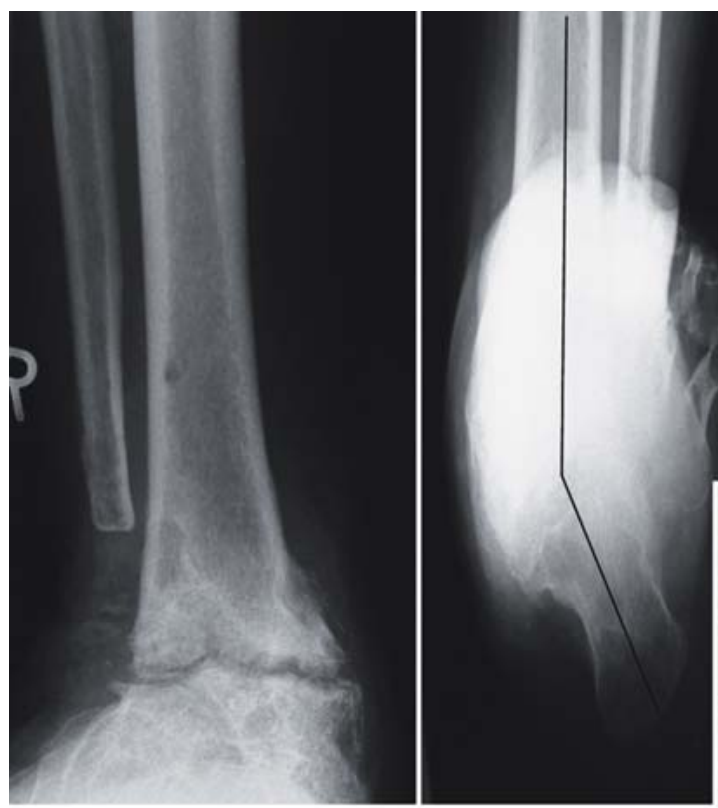

Abb.4a Rezidivpseudarthrose des OSG bei fixiertem Pes plano-valgus mit fortgeschrittener USG-Arthrose.
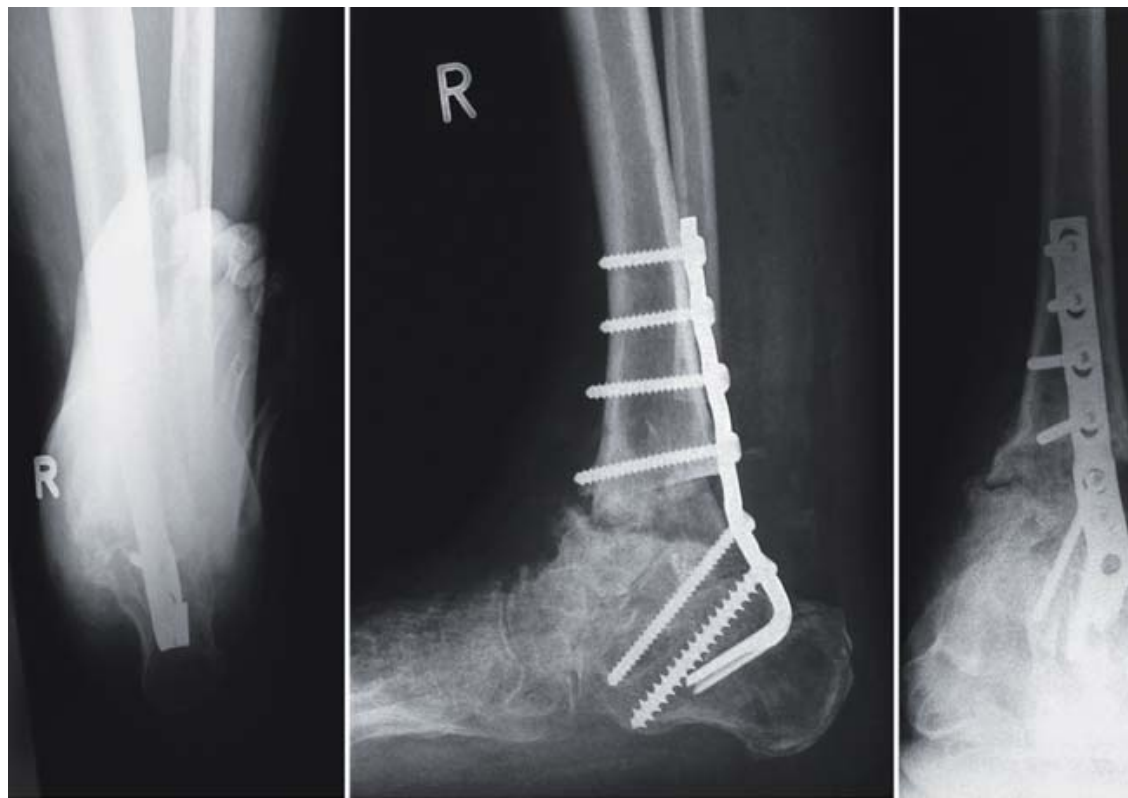

Abb. 4b Korrektur-Double-Arthrodese von OSG und USG mit jeweils autologem trikortikalem Beckenkammspan und kanülierter Winkelplatte. Radiologische Kontrolle postoperativ (links) und nach 4 Monaten postoperativ (rechts). Auch klinisch imponiert die Korrektur der Rückfußvalgusdeformität. 

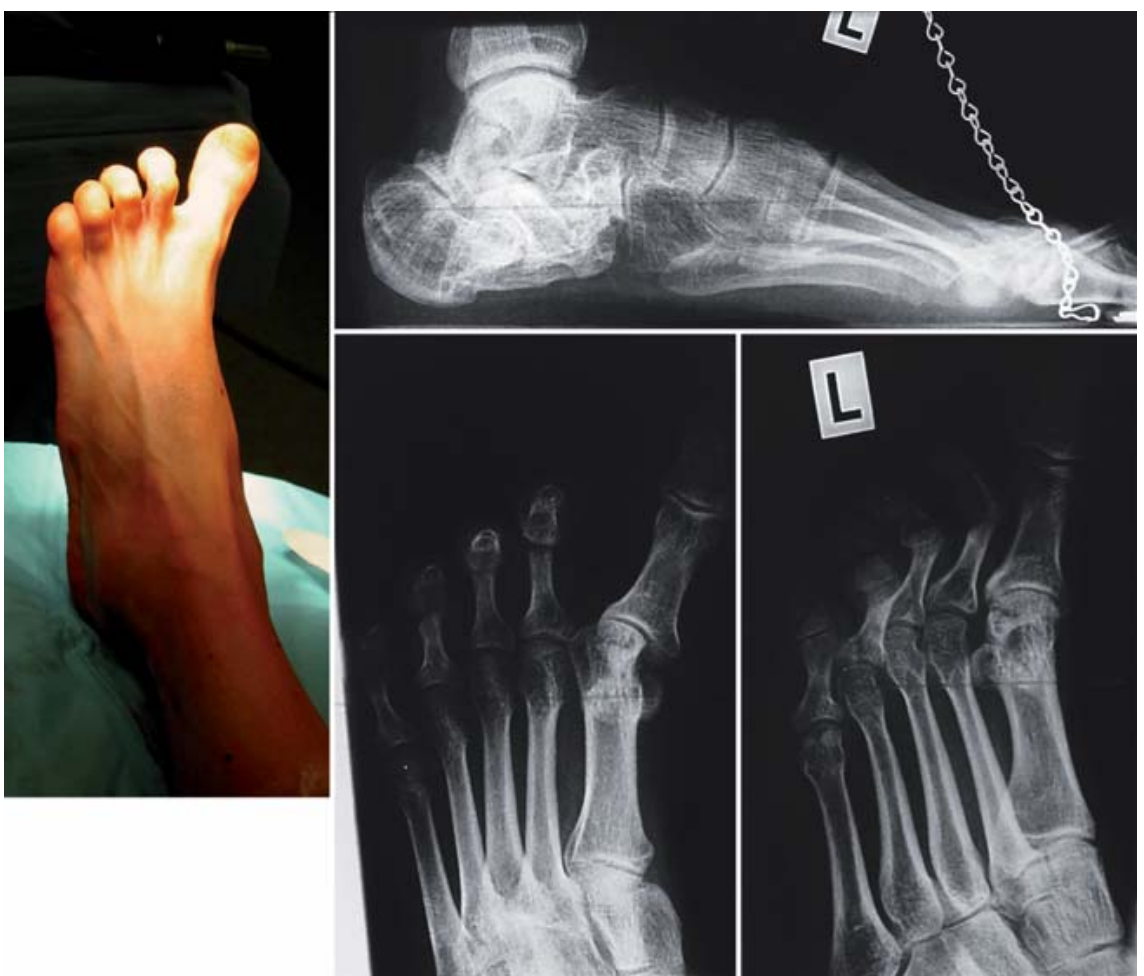
zehengrundgelenksluxation jeweils nach konservativer Behandlung/geschlossener Reposition und Ausbildung eines (unbehandelten) Fußkompartmentsyndroms.

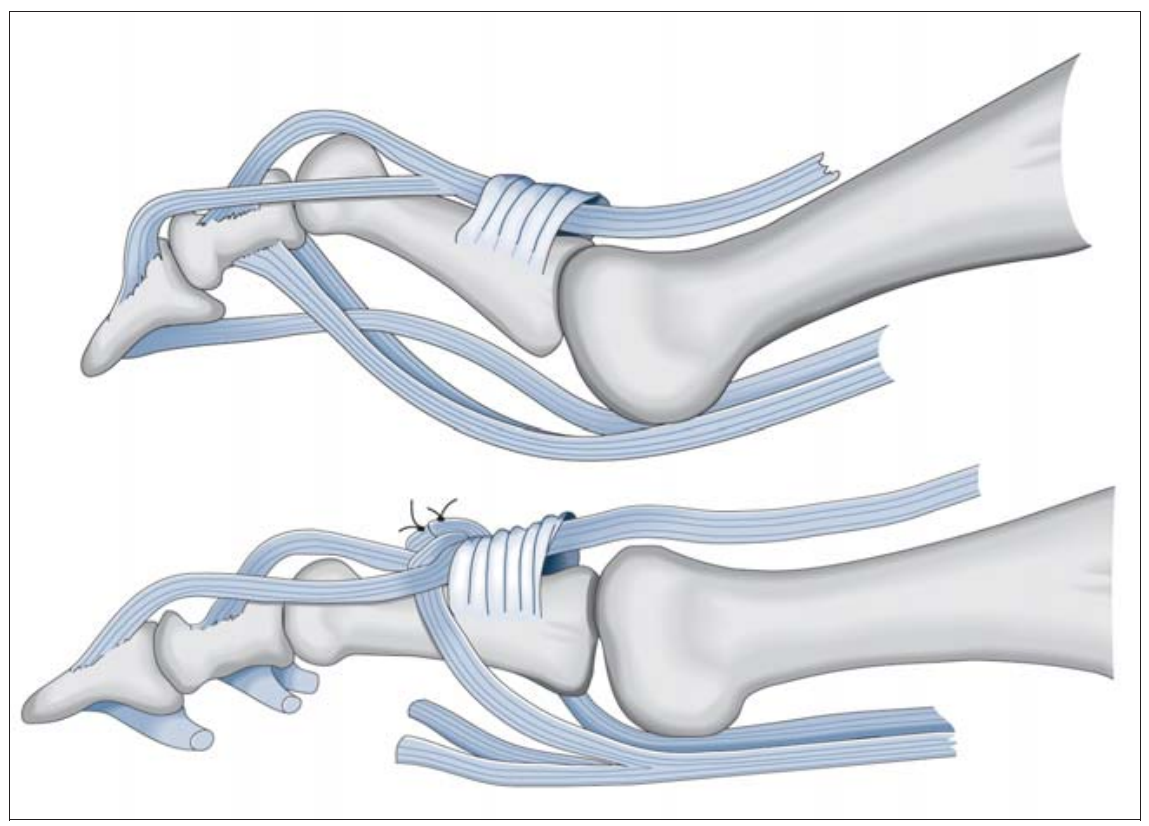

Abb.5b OP-Technik: Flexor-Extensor-Transfer bei flexiblen Krallenzehen.

terte laterale Wand, die ein Peronäalsehnenimpingement oder ein Verklemmen der Außenknöchelspitze bei Bewegungen verursacht, kann zudem bei diesem Eingriff beseitigt werden [8]. Die Applikation des Distraktors und die $\mathrm{Zu}-$ richtung des entsprechenden autologen trikortikalen Beckenkammspans richtet sich nach der jeweiligen Fehlstellung: Bei Valgusfehlstellung wird der Span lateral breiter als medial zugeschnitten (Abb. 2c). Bereits ohne Implantat resultiert nach Lösen des Distraktors ein guter Klemmsitz des Spans in der kor- rigierten Rückfußposition. Die Einbringung der Implantate (2 Schrauben genügen) von plantar ist vorteilhafter als jene von talar (Abb. 5c), da hier ein Impingement der Schraubenköpfe mit der anterioren Tibiakante und somit eine Limitierung der Extension im OSG droht.

Selbst bei erheblichem Ausmaß der primären Schädigung etwa bei der Kalkaneusluxationsfraktur ist es technisch einfacher zunächst eine Osteosynthese zur Wiederherstellung der Form des Rückfußes und dann sekundär eine subtalare Arthrodese durchzuführen (Abb. 3). Alternativ kann in begründeten Ausnahmesituationen eine einzeitige primäre Arthrodese sinnvoll sein, die allerdings zumeist ohne die zeitgleiche Osteosynthese des Fersenbeins nicht auskommt [6].

Die Stellung des Rückfußes und der Zustand des Subtalargelenks sollte grundsätzlich auch in die Analyse einbezogen werden, wenn hier primär gar keine Verletzung vorlag, aber eine relevante Fehlstellung (Abb.4): Eine wiederholt fehlgeschlagene OSG-Arthrodese bei vorbestehender massiver fixierter Plattfußdeformität kann durch einzeitige Korrektur der symptomatischen Fehlstellung im USG und der Pseudarthrose im Sinne der Double-Arthrodese angegangen werden. Auch hier ist eine ausschließliche Rekonstruktion in Bauchlage von posterolateral vorteilhaft; die entsprechenden knöchernen Defekte nach Anfrischen der Sklerosezone und Entfernung des Restknorpels im USG werden durch autologe trikortikale Beckenkammspäne aufgefüllt.

\section{Arthrodesen am Chopart-Gelenk}

Die erhebliche Varianz der Verletzungen im Chopart-Gelenk und ihrer möglichen Folgezustände und Fehlstellungsvarianten macht eine akribische Einzelfallprüfung erforderlich, inwieweit mehrdimensionale Fehlstellungen (z.B. auch Kombinationen mit rotatorischen Fehlstellungen) vorliegen und eine Arthrodese mehrerer Gelenke des Rückfußes zum Erzielen einer plantigraden Fußstellung erforderlich ist. Wenn es auch das Ziel ist, das Talonavikulargelenk als funktionelles Schlüsselgelenk der Fußwurzel zu erhalten [10,12], so bleibt oft bei massiver Gelenkdestruktion und/ oder avaskulärer Nekrose des Navikulare mit resultierender Fehlstellung keine andere Wahl als eine reorientierende, talonavikulare oder talokuneiforme 

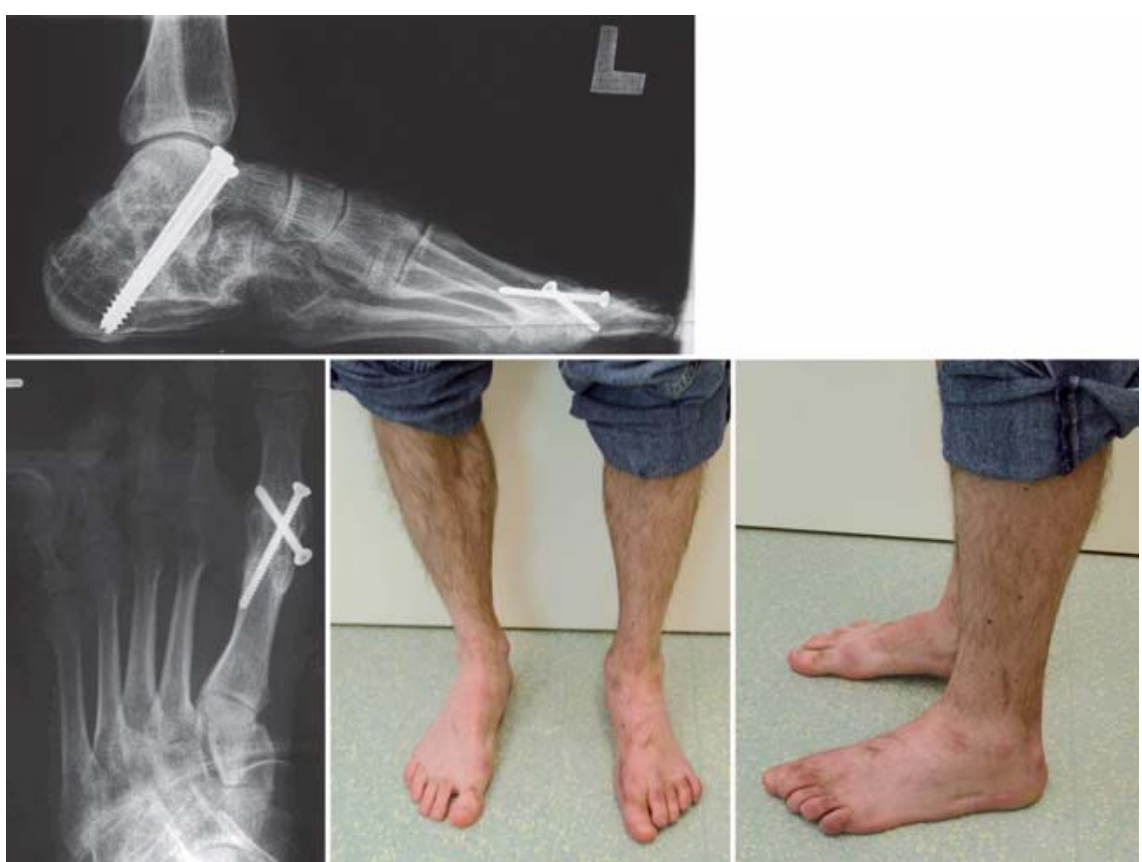

Abb.5c Postoperative Belastungsaufnahmen und klinisches Bild nach reorientierender Subtalargelenksarthrodese, Großzehengrundgelenksarthrodese und Klauenzehenkorrektur am 2.4. Strahl mit Resektionsarthroplastik und Flexor-Extensor-Transfer.

Arthrodese (Abb. 7) [3]. Das Ausmaß des Vorschadens und die große mit einem Span zu überbrückende Distanz bedingen jedoch lange Durchbauungszeiten. Die Osteosyntheseform sollte diesen Umständen Rechnung tragen. Gleichzeitige Übungsstabilität wird auch in dieser Region durch eine entsprechend stabile Versorgung mit winkelstabilen Implantaten gewährleistet, die auch im
Versagens- und Revisionsfall zum Einsatz kommen (Abb. 7 c).

Die Indikation für eine Tripelarthrodese bei posttraumatischer USG-Arthrose besteht selten, kann sich jedoch insbesondere bei kombinierten knöchernen Fehlstellungen und unfallbedingten Paresen ergeben [24], falls sich anderweitig keine plantigrade Fußposition erzielen lässt (Abb. 8). Im Regelfall ist hierzu ein Doppelzugang erforderlich. Die Implantatwahl wird einerseits durch die Knochenqualität, andererseits durch die lokale Weichteilsituation (Vermeidung auftragender Implantate) mitbestimmt. Sekundäre Fehlstellungen in Fußwurzel oder Vorfuß, die sich kompensatorisch bei lange bestehender traumatischer Deformität ergeben, sollten zweizeitig angegangen werden, da dann auch geprüft werden kann, inwieweit sich diese funktionell auswirken und überhaupt ein weiterer Korrekturbedarf besteht (Abb. 8c).

\section{Arthrodesen am Lisfranc-Gelenk}

Eine symptomatische posttraumatische Arthrose nach Lisfranc-Gelenkluxation oder Lisfranc-Gelenkfrakturen ist eine häufige Indikation zur Teilarthrodese des Lisfranc-Gelenks [16,25]. Typischerweise resultiert eine Arthrose nach rein ligamentärer Läsion häufiger als nach osteoligamentären Kombinationsverletzungen. Lediglich im US-amerikanischen Raum wird deshalb eine großzügige Indikation für eine primäre Arthrodese nach rein ligamentärer LisfrancGelenkverletzung befürwortet [23].

In jedem Fall ist eine Fehlstellung (meistens Abduktion des Vorfußes, Dorsaldislokation der Metatarsalia, schlussendlich entsteht ein posttraumatischer Plattfuß) in die Korrekturplanung einzubeziehen; somit bedarf es zumeist
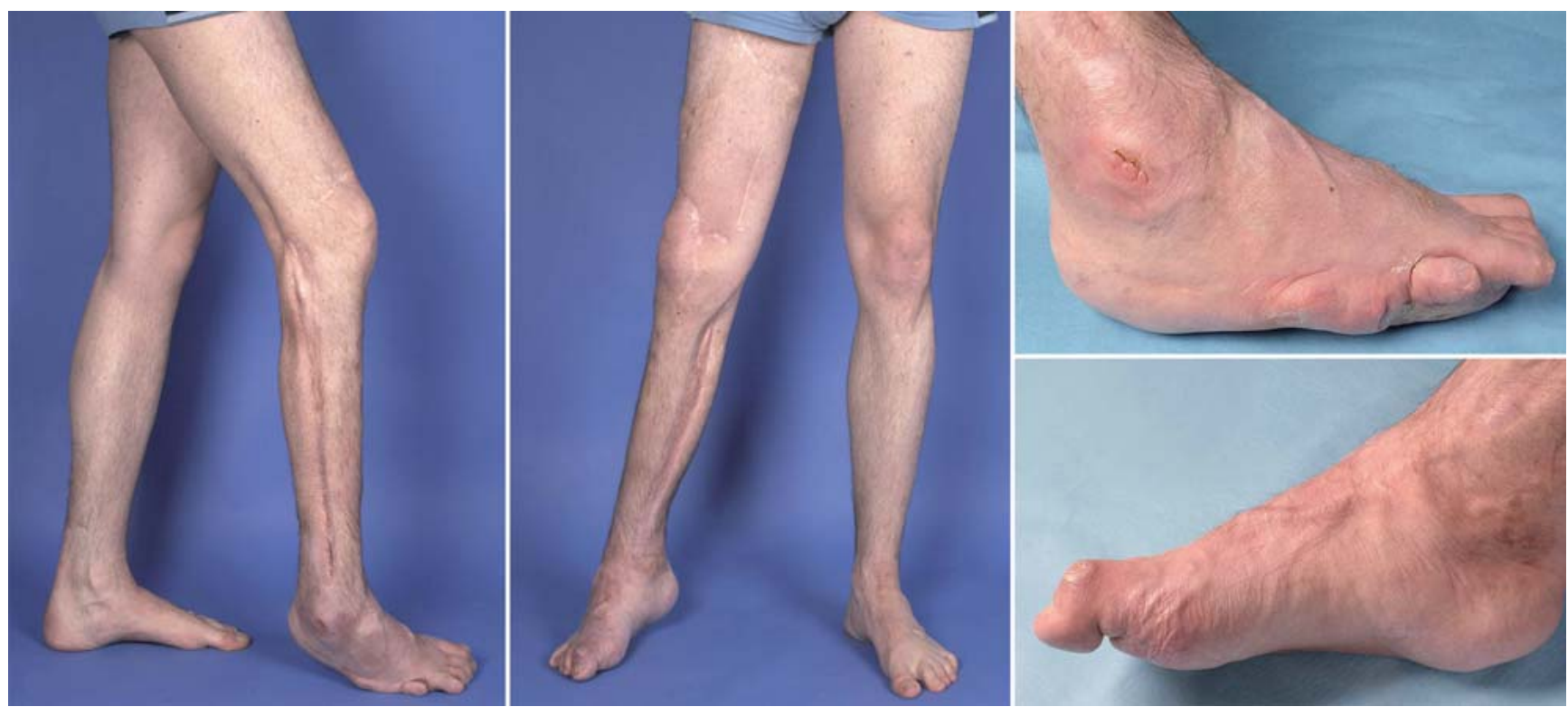

Abb. 6 a 25-jähriger Patient; 5 Jahre nach Unterschenkelkompartmentsyndrom bei Kniegelenksluxation mit Popliteaabriss und Läsion des N. tibialis. Ausbildung eines Pes equinovarus adductus mit Fistel am Außenknöchel bei chronischer Osteitis durch Druckulzerationen; Teilverlust der Metatarsalia 4 und 5 infolge chronischer Osteitiden. 

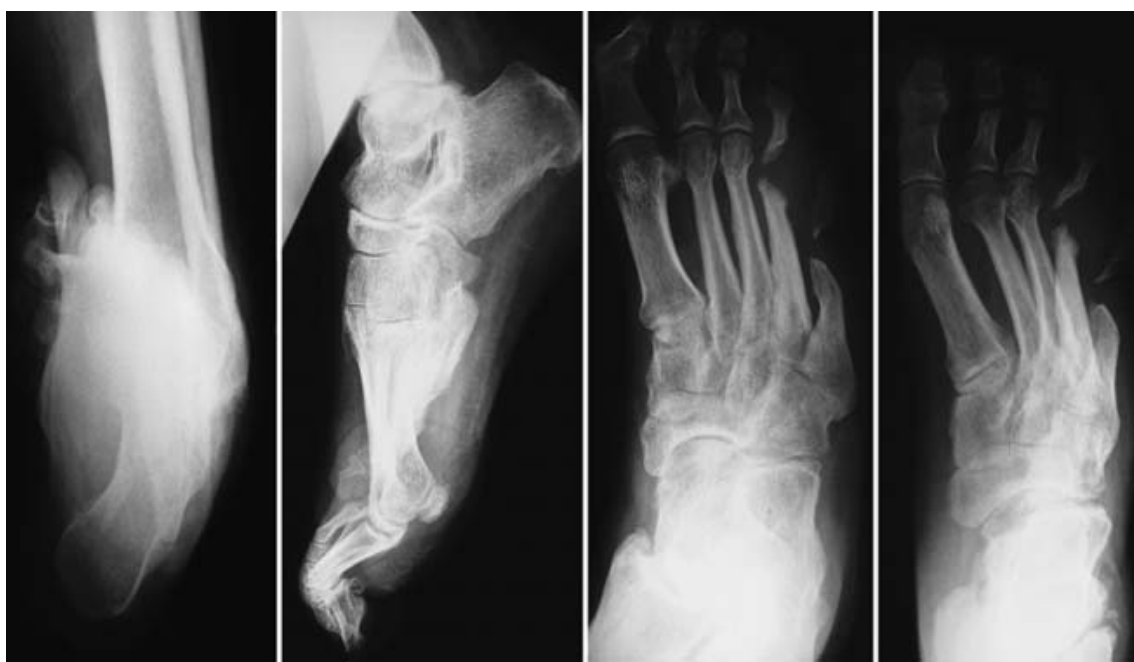

Abb.6b Standard-Röntgenbildserie (Saltzman, OSG/Fuß seitlich und a.-p. bzw. Fußwurzel schräg); korrekte Belastungsaufnahmen sind bei fehlender plantigrader Fußstellung nicht möglich.
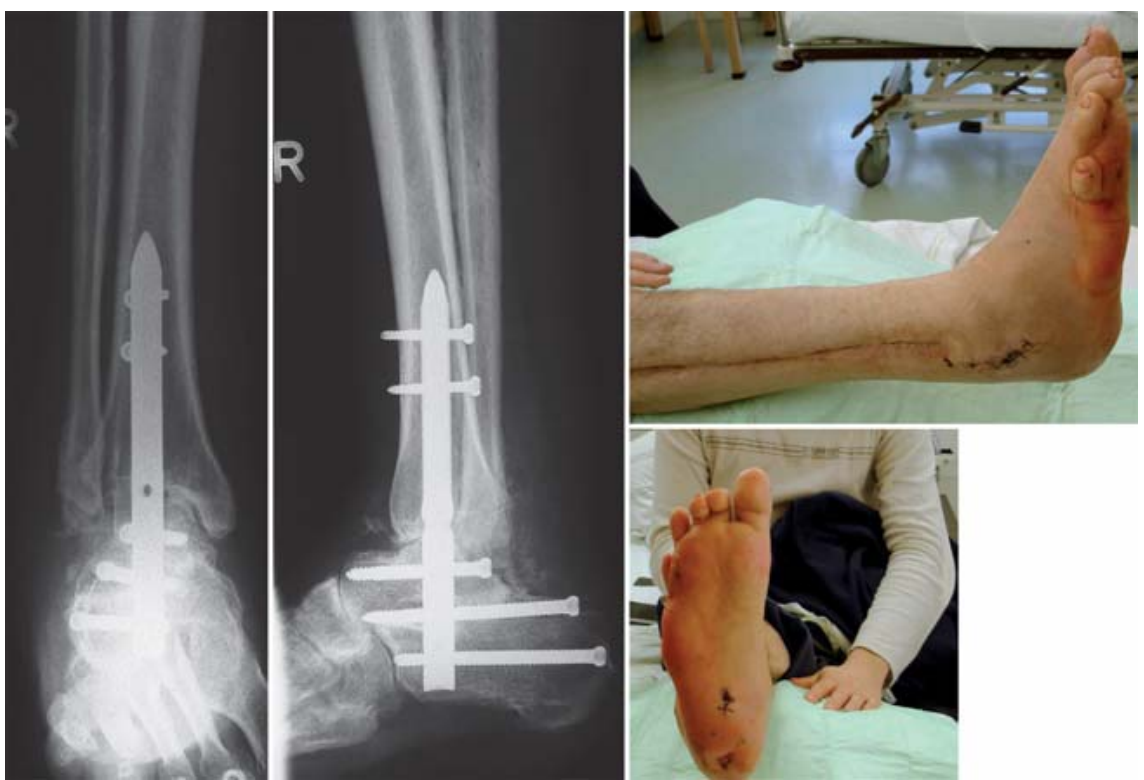

Abb.6c Postoperatives Resultat nach reorientierender Double-Arthrodese von OSG und USG mit retrogradem Verriegelungsmarknagel und Korrekturarthrodese des Großzehengrundgelenks nach Teilresektion der Fibula.
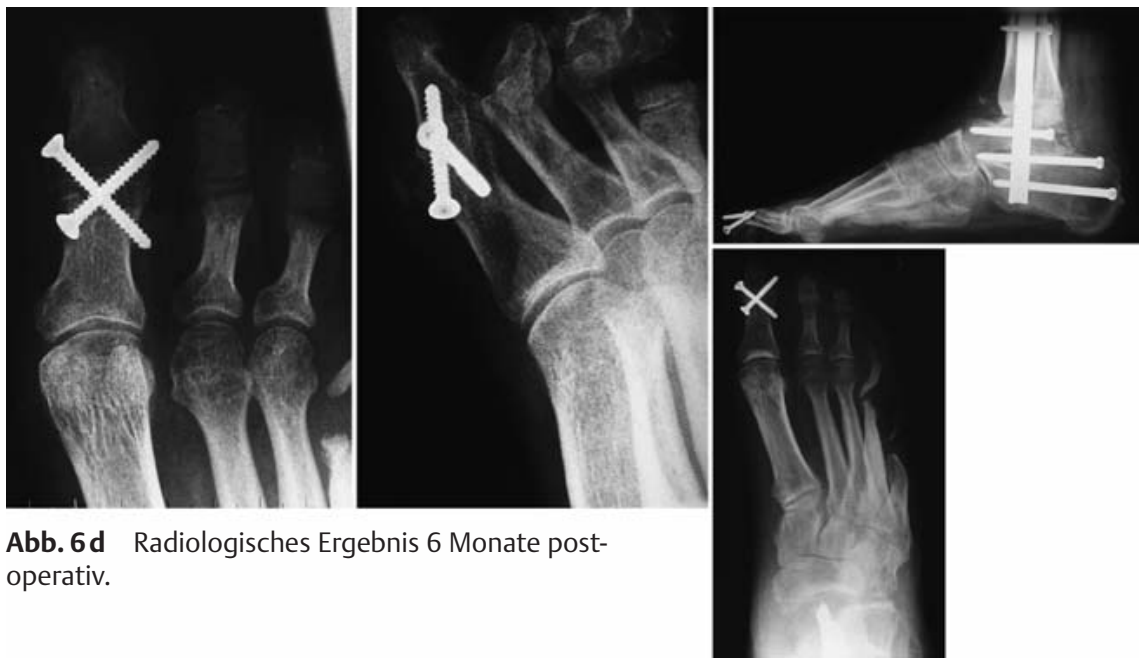

Abb. 6d Radiologisches Ergebnis 6 Monate postoperativ. der Auffüllung verbleibender Defekte nach Korrektur mit autologem Knochen. Wenn immer möglich, sollten die lateralen Lisfranc-Gelenke 4 und 5, deren hohe physiologische Mobilität für das Gangmuster entscheidend ist, erhalten bleiben und nicht in die Arthrodese einbezogen werden [12]. Als Implantate genügen im Regelfall 3,5-mm-Kortikalisschrauben $[16,23,25]$.

\section{Korrekturen am Vorfuß}

Die Notwendigkeit zur Korrektur ergibt sich zumeist nach Kettenverletzungen der Metatarsalia (z.B. Verheilung der Metatarsalia mit plantarer Dislokation der Köpfchen) oder bei symptomatischer Arthrose/fixierter Fehlstellung des MTP-I-Gelenks (Abb. 5 und 6). Abweichungen in der Horizontalebene wirken sich meist nicht so ungünstig aus wie Fehlstellungen in der Sagittalen (oftmals auch mit einer Verkürzung der jeweiligen Metatarsalstrahlen vergesellschaftet), die zumeist in eine Metatarsalgie münden [26].

Das Ausmaß der Gelenkdestruktion und das Potenzial einer Weichteilkorrektur entscheidet darüber, ob ein Gelenkerhalt möglich ist. Eine Großzehengrundgelenksarthrodese (das Großzehengrundgelenk ist kein essenzielles Gelenk! ) bietet auch beim jüngeren Patienten die Chance für eine gute Gehfunktion.

Allein resezierende Maßnahmen an den Zehen 2 bis 5 bei Krallen- und Hammerzehen durch Weichteilkontrakturen, etwa nach Fußkompartmentsyndrom, resultieren zumeist in dorsal subluxierten instabilen und funktionslosen $\mathrm{Ze}$ henstrahlen mit Schuhkonflikt. Hier bietet sich eine der zahlreichen Modifikationen des Flexor-Extensor-Sehnentransfers (Abb.5b und c) an, eine Zehenstellungskorrektur mit bleibendem Bodenkontakt zu erzielen $[12,19]$. Sparsame Teilresektionen des Grundgelenks und/oder metatarsale Verkürzungsosteotomien können auch vorteilhafterweise mit einem Balancing der Beugeund Strecksehnen kombiniert werden, um eine funktionierende Einheit aus Metatarsale und Zehe wiederherzustellen (Abb. 5 c) [12].

\section{Tibio-talokalkaneare Arthrodese}

Eine kombinierte korrigierende OSGund Subtalargelenkarthrodese wird im Regelfall bei schwerer komplexer Rück- 


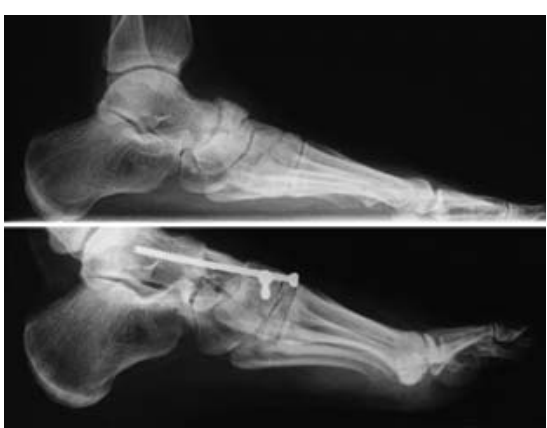

Abb.7a Posttraumatische Nekrose des Os naviculare mit Sinterung und Verkürzung der medialen Fußsäule. Korrekturarthrodese mit autologem trikortikalen Beckenkammspan und talokuneiformer Arthrodese mit Kleinfragmentzugschrauben.

fußdeformität mit gleichzeitiger Destruktion beider Gelenkkompartimente und/oder symptomatischer bifokaler Arthrose am Rückfuß erwogen. Darüber hinaus ist eine tibio-talokalkaneare Korrekturarthrodese ein typisches SalvageVerfahren nach fehlgeschlagenen Voreingriffen ([9,11] Abb.4). Vor einer zunehmenden Verbreitung der retrograden Verriegelungsmarknagelung zur USG- und OSG-Arthrodese aufgrund der Kriterien einer minimalinvasiven Implantationstechnik und einer hohen Primärstabilität kann bei Intaktheit des USG nur gewarnt werden. Nach DoubleArthrodese kann grundsätzlich keine normale Gehfunktion resultieren.

Die Frage nach dem geeigneten Implantat ist prinzipiell dem Ziel - einer plantigraden Fußstellung und einem korrekten Rückfußalignement - nachgeordnet.

Eine tibio-talokalkaneare Arthrodese kann zentrales Element einer Korrektur einer komplexen Fehlstellung sein, die weiterer Maßnahmen (Weichteilbalancing) bedarf (Abb. 6).

Entsprechend der individuellen Situation ist die präoperative Planung der einzelnen Korrekturschritte ganz wesentlich. Bei Verwendung der retrograden Nagelungstechnik ist die Wahl des Eintrittspunktes ein entscheidender Schritt der Operation, da hierdurch die Position im Fersenbein und die Rückfußstellung festgelegt werden. Die anatomische Achsausrichtung des Kalkaneus, der lateral unter dem Talus und Tibiaschaft zu liegen kommt, macht es bei geraden Nageltypen erforderlich, bei zentralem Nageleintrittspunkt relativ nahe an der medialen Kalkaneuswand

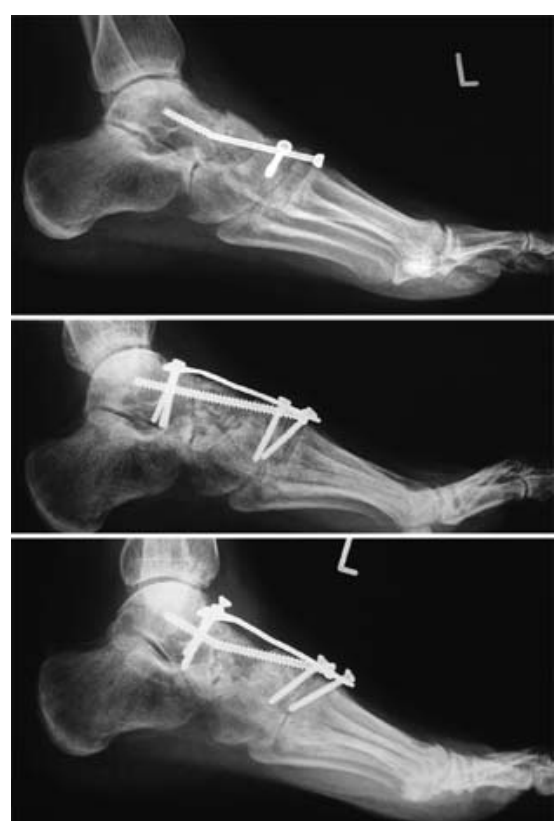

Abb. 7b Implantatbruch vor Ausheilung. Revision mit erneuter autologer Span-Spongiosaplastik und Rearthrodese mit Kleinfragment-T-Plättchen und Plattenlockerung proximal bei ausbleibender Spanintegration distal.

nach proximal in den Talus vorzudringen oder aber eine Verkippung des Kalkaneus in Varusstellung zu riskieren [11]. Verbleibende knöcherne Defekte sollten in jedem Fall mit autologem Knochen aufgefüllt werden (Abb. 8).

\section{Kombinierte Eingriffe: reorientieren- de Arthrodesen - Sehnentransfers}

Traumatisch bedingte ossäre Fehlstellungen werden durch Paresen (z. B. Peronäusparese) oft ungünstig beeinflusst und verstärkt. Sind nach gründlicher klinischer Untersuchung, ggf. ergänzt durch eine differenzierte neurologische Untersuchung, andere Muskelgruppen des Fußes gut funktionsfähig, besteht die Option eines Sehnentransfers oder der Kombination aus knöchernem Korrektureingriff mit gleichzeitigem Sehnentransfer $[10,12,17,19,26]$. Hierfür bedarf es ausreichender Erfahrung, um durch geeignete Transferwahl (ausreichende Kraftentfaltung für die vorgesehene Funktion) und die intraoperativen Parameter (etwa die Vorspannung) das spätere Resultat vorwegzunehmen (vgl. Abb.9). Bei dieser Art von Eingriff besteht einerseits das größte Potenzial vorhandene Restfunktionen optimal zu nutzen, andererseits aber auch das größte Risiko, das angestrebte Korrekturziel zu verfehlen oder in einer erneuten Fehlstellung zu enden.

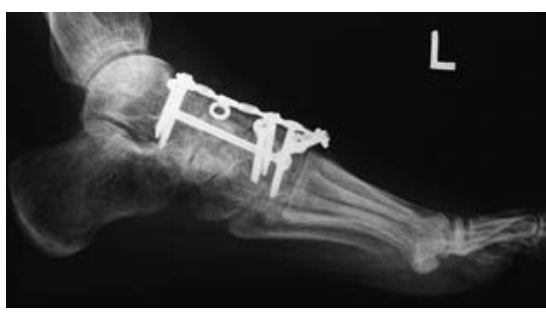

Abb.7c Revision mittels winkelstabiler Kleinfragmentplatte und Ausheilung in korrekter Länge der medialen Fußsäule.

\section{Tibiokalkaneare Arthrodese}

Extraanatomische Verfahren wie die tibiokalkaneare Arthrodese sind ausschließlich Salvage-Verfahren, die eine Berechtigung als Alternative zu einer Amputation besitzen [18]. Bei Ausheilung resultiert zwar häufig eine deutliche Beinlängenverkürzung von etwa $2-5 \mathrm{~cm}$, der Erhalt des Chopart-Gelenks lässt jedoch eine Restbeweglichkeit in der Sagittalebene von bis zu 20 Grad zu. Zugrunde liegt meist ein subtotaler Verlust des Talus nach posttraumatischer Osteonekrose/Infekt, wobei die lokale Situation einen Wiederaufbau der Talushöhe, etwa durch einen entsprechend dimensionierten trikortikalen Beckenkammspan, nicht ratsam erscheinen lässt.

Bei floridem Infekt bleibt als Standardtechnik nach radikalem Débridement zumeist der Fixateur extern, z.B. als Ringfixateur mit hoher Stabilität, das Verfahren der Wahl zur Einstellung einer plantigraden Fußstellung [17]. Ein wesentlicher Vorteil liegt in der Option der frühzeitigen Vollbelastbarkeit nach Konsolidierung der Weichteilsituation.

Liegt kein tiefer Infekt der Indikation zur tibiokalkanearen Fusion zugrunde, können auch kanülierte Winkelplatten $(4,5 \mathrm{~mm})$ mit gutem Erfolg anstatt eines Ilizarov-Ringfixateurs zur Arthrodese genutzt werden.

\section{Schlussfolgerung}

Vor einer posttraumatischen Korrektur am Fuß bei manifester Pseudarthrose, posttraumatischer Arthrose und/oder Fehlstellung bzw. Paresen bedarf es einer akribischen Analyse der zugrunde liegenden strukturellen und funktionellen Störung. 

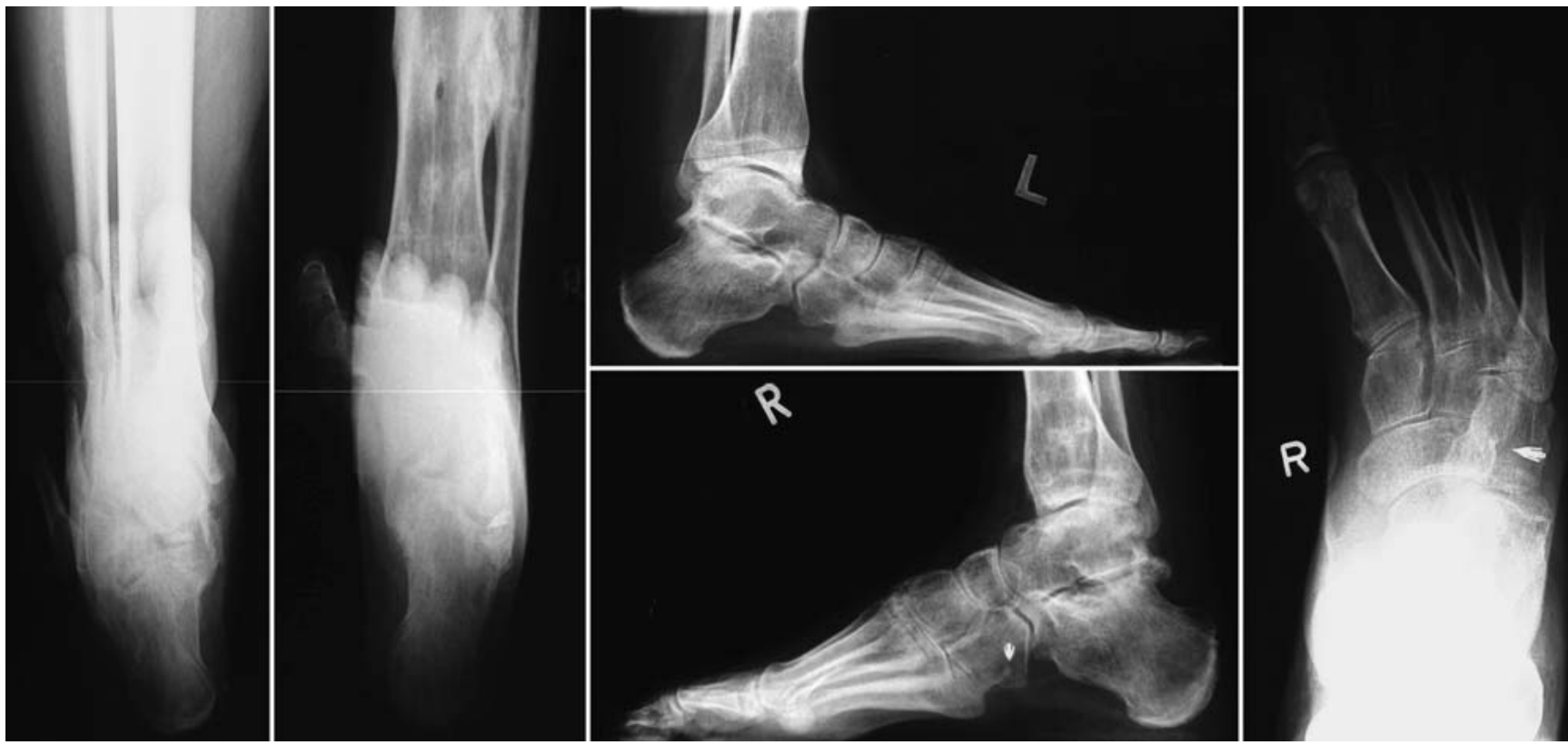

Abb. 8 a Z.n. Komplextrauma mit Pes cavovarus adductus. Fehlgeschlagener Sehnentransfer auf die laterale Fußsäule bei Peronäusparese.

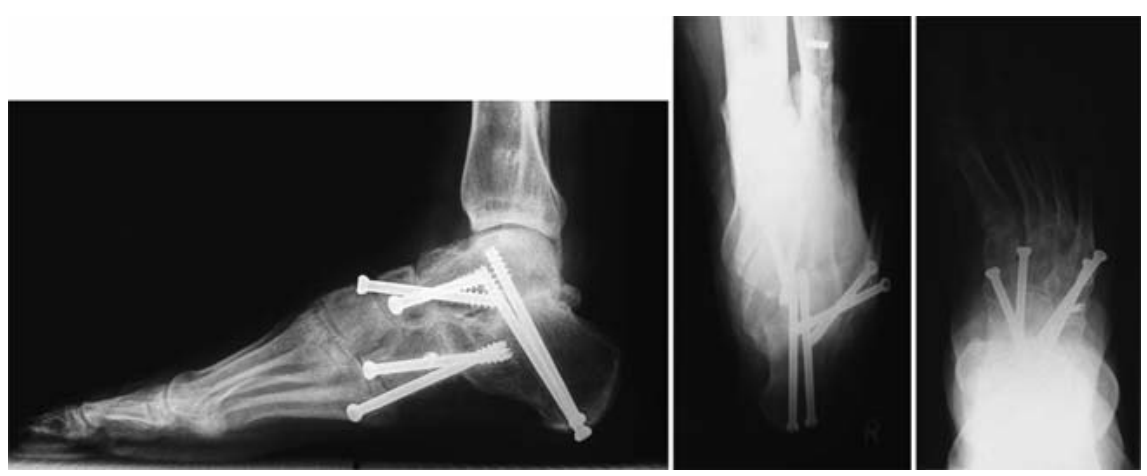

Abb. $\mathbf{8 b}$ Tripelkorrekturarthrodese des USG. Es verbleibt eine residuale geringe Adduktion distal der Korrekturebene im Vorfuß.

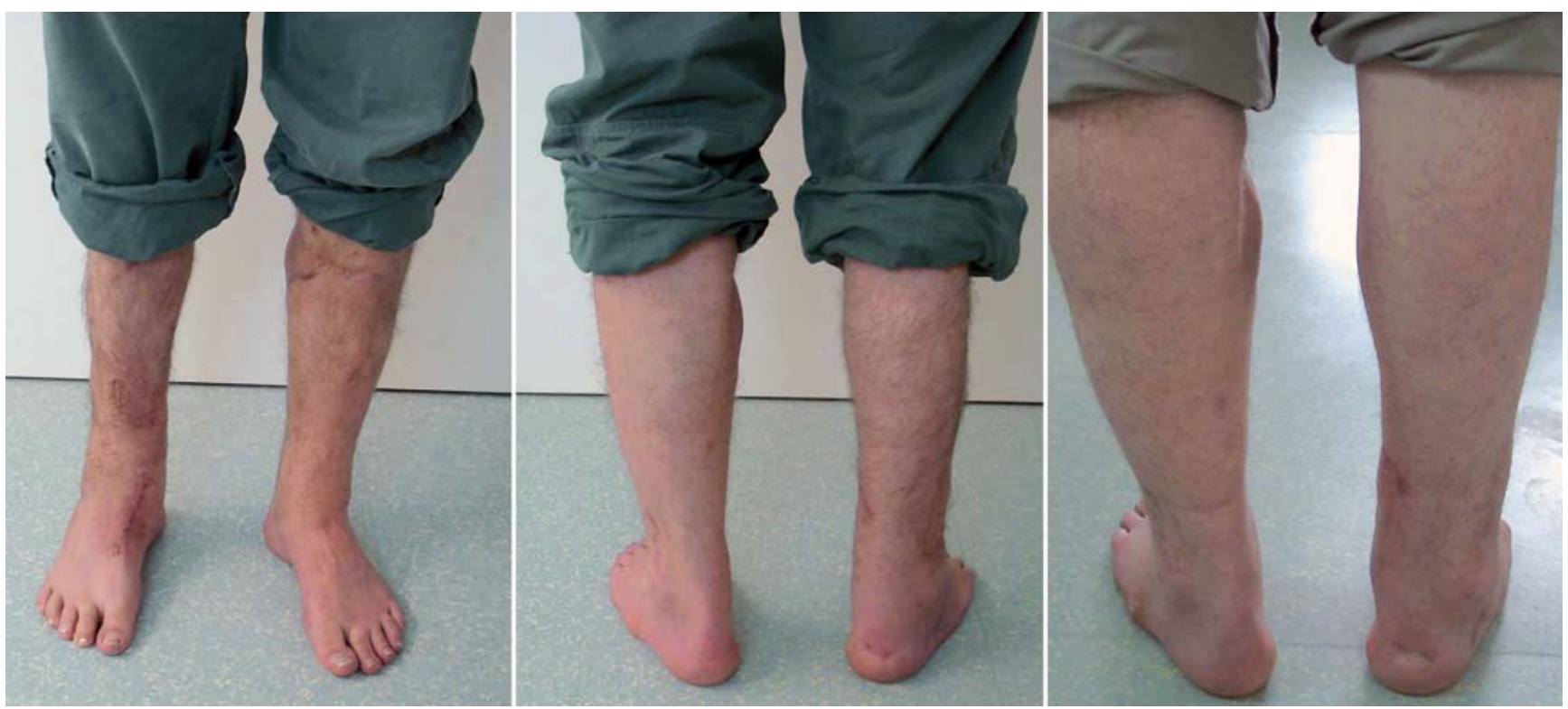

Abb. 8c Resultat 9 Monate postoperativ. Der Patient benötigt kein orthopädisches Schuhwerk. 


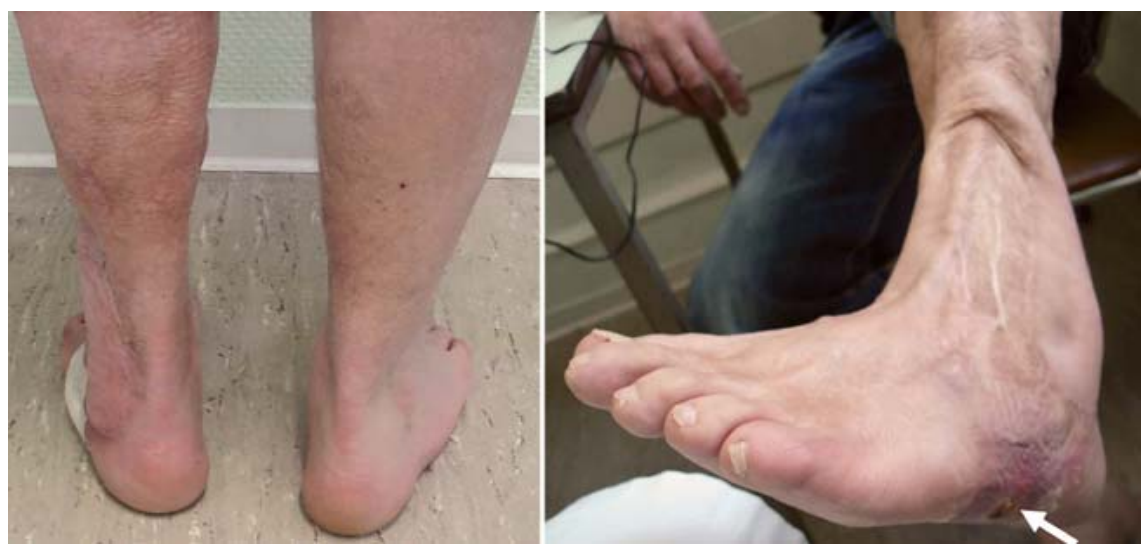

Abb.9a 36-jähriger Mann 7 Jahre nach Komplextrauma des Fußes und Weichteildeckung mit freiem Radialislappen. Bei fehlender Funktion der Peronäalmuskulatur kommt es zur Rückfußvarusdeformität und persistierenden Ulkusbildung unter dem proximalen Metatarsale 5 .
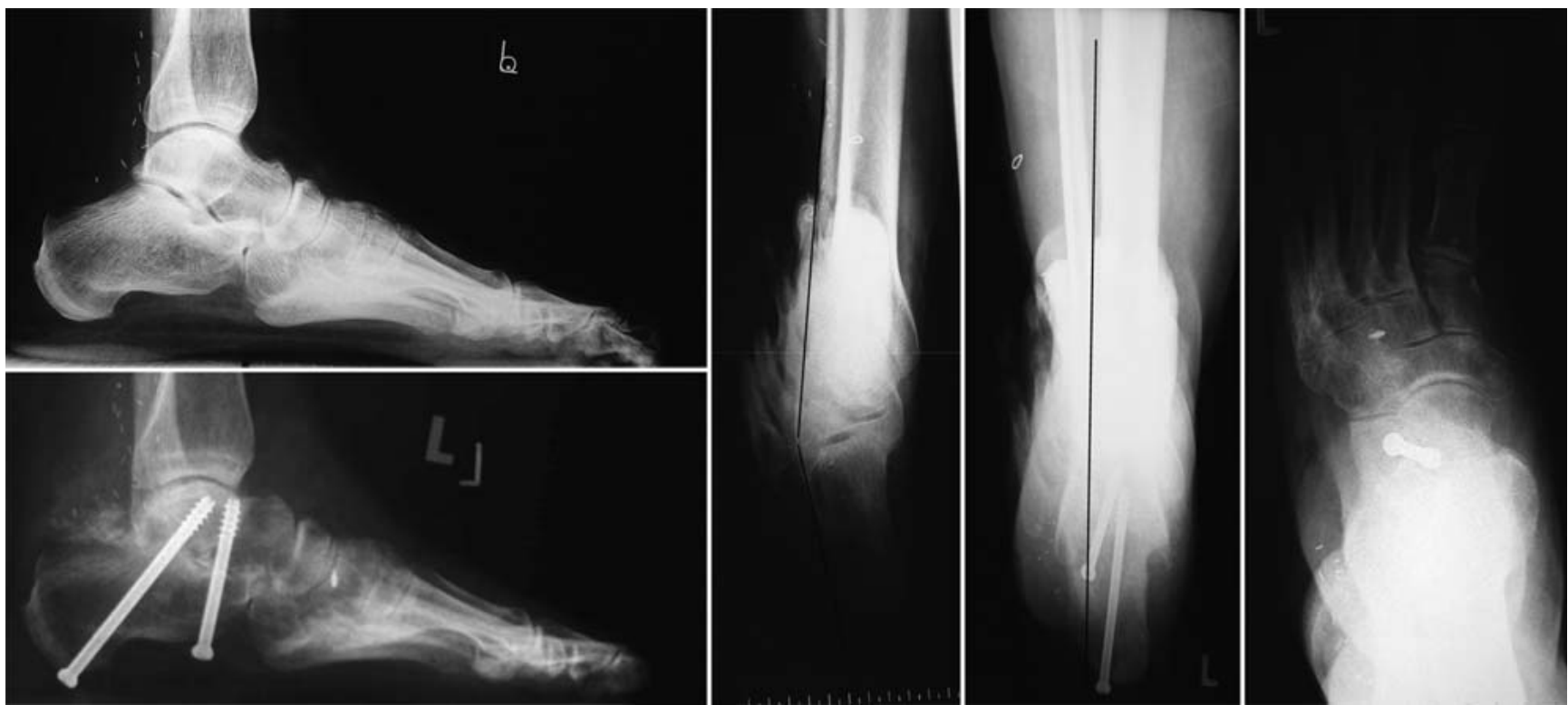

Abb.9b Reorientierende subtalare Schraubenarthrodese und Transfer der halben Tibialis-anterior-Sehne auf das Os cuneiforme laterale.
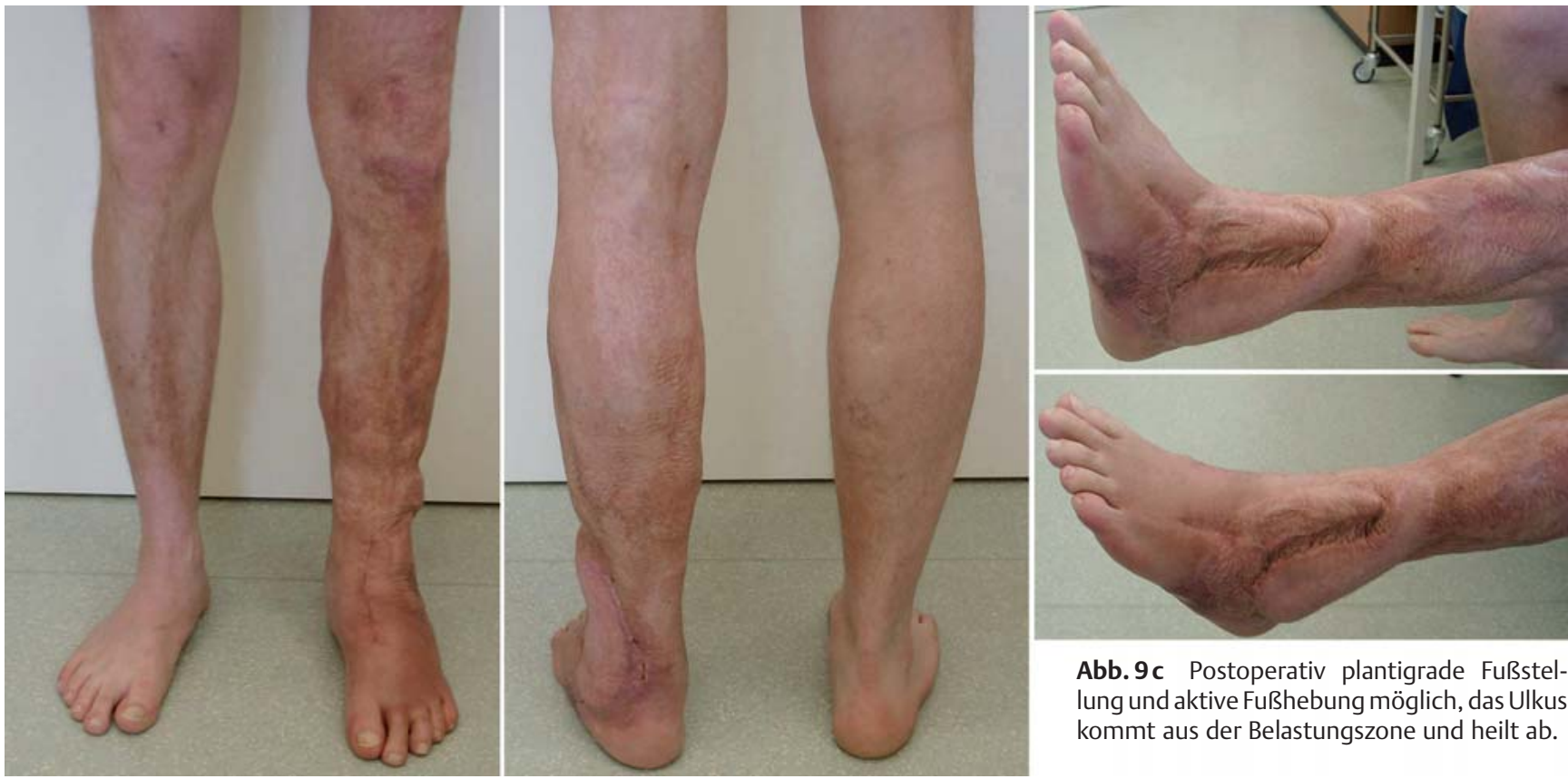

Abb.9c Postoperativ plantigrade Fußstellung und aktive Fußhebung möglich, das Ulkus kommt aus der Belastungszone und heilt ab. 
Das Ergebnis der Recherche sollte ein Behandlungskonzept sein, das unter geringst möglichem therapeutischem $\mathrm{Ri}$ siko die Ziele eines plantigraden Fußes mit korrekten Achsverhältnissen umzusetzen erlaubt. Ferner ist das Konzept mit den Erwartungen des Patienten abzugleichen, der sich zumeist eine Besserung der Gehfunktion bei Beseitigung bzw. Minimierung seiner Schmerzen wünscht.

Das Portfolio der Korrekturoptionen am Fuß umfasst Osteotomien, Knochenspan-Spongiosaplastiken, reorientierende Arthrodesen und Sehnentransfers. Die Zahl der zu arthrodesierenden Gelenke sollte auf ein notwendiges Minimum beschränkt werden.

\section{Literatur}

${ }^{1}$ Astion DJ, Deland JT, Otis JC, Kenneally S. Motion of the hindfoot after simulated arthrodesis. J Bone Joint Surg [Am] 1997; 79: 241 246

2 Bibbo C, Anderson RB, Davis WH. Complications of midfoot and hindfoot arthrodesis. Clin Orthop 2001; 391: 45-58

${ }^{3}$ Chen $\mathrm{CH}$, Huang PJ, Chen TB, Cheng YM, Lin SY, Chiang HC, Chen LC. Isolated talonavicular arthrodesis for talonavicular arthritis. Foot Ankle Int 2001; 22: 633-636

${ }^{4}$ Clare MP, Lee WE, Sanders RW. Intermediate to long-term results of a treatment protocol for calcaneal fracture malunions. J Bone Joint Surg [Am] 2005; 87: 963-973

${ }^{5}$ Csizy M, Buckley R, Tough S, Leighton R, Smith J, McCormack R, Pate G, Petrie D, Galpin R. Displaced intra-articular calcaneal fractures. Variables predicting late subtalar fusion. J Orthop Trauma 2003; 17: 106-112
${ }^{6}$ Easley ME, Trnka HJ, Schon LC, Myerson MS. Isolated subtalar arthrodesis. J Bone Joint Surg [Am] 2000; 82: 613-624

7 Flemister AS, Infante AF, Sanders RW, Walling AK. Subtalar arthrodesis for complications of intra-articular calcaneal fractures. Foot Ankle Int 2000; 21: 392 - 399

8 Grass R, Zwipp H. Die subtalare Korrekturarthrodese nach fehlerhaft verheilter Fersenbeinfraktur. Operat Orthop Traumatol 2000; 2: $316-327$

9 Grass R, Rammelt S, Heineck J, Zwipp H. Die Rückfußarthrodese durch retrograde Marknagelung. Orthopäde 2005; 34: 1238 - 1244

10 Klaue K, Hansen ST. Principles of surgical reconstruction of the mid- and hindfoot. Eur J Foot Ankle Surg 1994; 1: $37-44$

11 Hammett R, Hepple S, Forster B, Winson I. Tibiotalocalcaneal (hindfoot) arthrodesis by retrograde intramedullary nailing using a curved locking nail. The result of 52 procedures. Foot Ankle Int 2005; 26: 810 - 815

12 Hansen ST. Functional Reconstruction of the Foot and Ankle. Philadelphia: Lippincott, Williams \& Wilkins, 2000

13 Joseph TN, Myerson MS. Correction of multiplanar hindfoot deformity with osteotomy, arthrodesis, and internal fixation. Instr Course Lect 2005; 54: 269-276

14 Lamm BM, Paley D. Deformity correction planning for hindfoot, ankle and lower limb. Clin Podiatr Med Surg 2004; 21: 305- 326

${ }_{15}$ Mittlmeier T, Klaue K, Beck M. Posttraumatische Korrekturen am Rückfuß. Unfallchirurg 2006; 109: 125-148

${ }^{16}$ Mulier T, Reynders P, Dereymaeker G et al. Severe Lisfrancs Injuries: primary arthrodesis or ORIF? Foot Ankle Int 2002; 23: $902-$ 905

17 Myerson MS (Ed). Foot and Ankle Disorders. Philadelphia: W.B. Saunders, 2000

18 Myerson MS, Alvarez RG, Lam PW. Tibiocalcaneal arthrodesis for the management of severe ankle and hindfoot deformities. Foot Ankle Int 2000; 21: 643-650

19 Pisani G. Fußchirurgie. Stuttgart, New York: Thieme, 1998
${ }^{20}$ Rammelt S, Grass R, Schikore H, Zwipp H. Verletzungen des Chopart-Gelenks. Unfallchirurg 2000; 105: 371 - 385

${ }^{21}$ Rammelt S, Grass R, Zawadski T, Biewener A, Zwipp H. Foot function after subtalar distraction bone-block arthrodesis. J Bone Joint Surg [Br] 2004; 86: 659-668

22 Saltzman CL, El-Khoury GY. The hindfoot alignment view. Foot Ankle Int 1995; 16: $572-576$

${ }^{23}$ Sangeorzan BJ, Veith RG, Hansen Jr ST. Salvage of Lisfranc's tarsometatarsal joint by arthrodesis. Foot Ankle Int 19990; 10: $193-$ 200

${ }^{24}$ Weber M, Schwer H, Zilkens KW, Siebert CH. Tibio-calcaneo-naviculo-cuboidal arthrodesis: 6 patients followed for $1-8$ years. Acta Orthop Scand 2002; 73: 98-103

${ }^{25}$ Zwipp H. Chirurgie des Fußes. Wien, New York: Springer, 1994

${ }^{26}$ Zwipp H, Rammelt S. Posttraumatische Korrekturen am Fuß. Zentralbl Chir 2003; 128: $218-226$

\section{Univ.-Prof. Dr. med. Thomas Mittlmeier \\ Klinikdirektor \\ Dr. med. Markus Beck \\ Leitender Oberarzt}

Abteilung für Unfall- und Wiederherstellungschirurgie Chirurgische Klinik und Poliklinik der Universität Rostock Schillingallee 35 18055 Rostock 